\title{
Lewis Acidity and Basicity: Another Measure of Carbene Reactivity
}

Trent H. Stein, Monica Vasiliu, Anthony J. Arduengo, III, and David A. Dixon*,†

Department of Chemistry and Biochemistry, Shelby Hall, The University of Alabama, Box

870336, Tuscaloosa, AL 35487-0336.

Ref. 33: Frisch, M. J.; Trucks, G. W.; Schlegel, H. B.; Scuseria, G. E.; Robb, M. A.; Cheeseman, J. R.; Scalmani, G.; Barone, V.; Petersson, G. A.; Nakatsuji, H.; Li, X.; Caricato, M.; Marenich, A. V.; Bloino, J.; Janesko, B. G.; Gomperts, R.; Mennucci, B.; Hratchian, H. P.; Ortiz, J. V.; Izmaylov, A. F.; Sonnenberg, J. L.; Williams-Young, D.; Ding, F.; Lipparini, F.; Egidi, F.; Goings, J.; Peng, B.; Petrone, A.; Henderson, T.; Ranasinghe, D.; Zakrzewski, V. G.; Gao, J.; Rega, N.; Zheng, G.; Liang, W.; Hada, M.; Ehara, M.; Toyota, K.; Fukuda, R.; Hasegawa, J.; Ishida, M.; Nakajima, T.; Honda, Y.; Kitao, O.; Nakai, H.; Vreven, T.; Throssell, K.; Montgomery, J. A., Jr.; Peralta, J. E.; Ogliaro, F.; Bearpark, M. J.; Heyd, J. J.; Brothers, E. N.; Kudin, K. N.; Staroverov, V. N.; Keith, T. A.; Kobayashi, R.; Normand, J.; Raghavachari, K.; Rendell, A. P.; Burant, J. C.; Iyengar, S. S.; Tomasi, J.; Cossi, M.; Millam, J. M.; Klene, M.; Adamo, C.; Cammi, R.; Ochterski, J. W.; Martin, R. L.; Morokuma, K.; Farkas, O.; Foresman, J. B.; Fox, D. J. Gaussian 16, Revision A.03, Gaussian, Inc., Wallingford CT, 2016.

†dadixon@ua.edu 
Table S1. G3MP2 total energies in Hartree.

\begin{tabular}{|c|c|c|c|c|}
\hline \multirow[t]{2}{*}{ No } & \multicolumn{2}{|l|}{ Fluorinated } & \multicolumn{2}{|l|}{ Protonated } \\
\hline & $\Delta \mathrm{H}_{0 \mathrm{~K}}$ & $\Delta \mathrm{H}_{298 \mathrm{~K}}$ & $\Delta \mathrm{H}_{0 \mathrm{~K}}$ & $\Delta \mathrm{H}_{298 \mathrm{~K}}$ \\
\hline 1 & -325.569569 & 25.563430 & -226.225309 & 26.220521 \\
\hline 2 & 58 & 8 & & 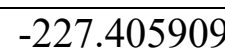 \\
\hline 3 & & & & \\
\hline 4 & -405.230032 & -405.220546 & 305.882152 & -305.873070 \\
\hline 5 & -404.051368 & -404.041978 & 8866 & -304.7 \\
\hline 6 & -482.5 & -482.494619 & 1634 & -383.18 \\
\hline 7 & -365.317383 & -365.311609 & -265.877180 & -265.872624 \\
\hline 8 & -1010.575921 & -1010.569136 & 9916 & -911. \\
\hline 9 & -255.41 & -255 & 505 & -155 \\
\hline 10 & -254.2 & & & \\
\hline 11 & & & & \\
\hline 12 & -212 & -214 & & 719 \\
\hline 13 & -138. & -138 & & -39 \\
\hline 14 & -238.1 & $-238 .($ & 438 & -138 \\
\hline 15 & -337. & -337. & 507 & -237 \\
\hline 16 & -178.1 & -178 & & -78.6 \\
\hline 17 & -217 & 61 & 441 & -117 \\
\hline 18 & -29 & -2 & & $-19 t$ \\
\hline 19 & -475 & & & \\
\hline 20 & -81 & -8 & & -71 \\
\hline 21 & -42 & 14 & 40 & -325 \\
\hline 22 & -719 & -719 & 238 & -620 \\
\hline 23 & -546. & -546. & 016 & -447 \\
\hline 24 & -954. & -954. & & -855 . \\
\hline 25 & -252.080416 & -252.074621 & 628 & -152.563068 \\
\hline 26 & -291.414359 & -291.407863 & -191 & -191.855604 \\
\hline 27 & -1057 & -1057 & 33 & -957. \\
\hline 28 & & -6 & & -59 \\
\hline 29 & & 0 & & 308 \\
\hline 30 & -330 & 27 & 384 & -230 \\
\hline 31 & -33 & -3 & 570 & -230 \\
\hline 32 & -323.269000 & -323.263189 & 717 & -223.618662 \\
\hline 33 & -323.189153 & -323.182433 & -223.598708 & -223.592301 \\
\hline 34 & -306. & -306.246341 & -206.720775 & -206.715652 \\
\hline 35 & -289.2 & -289.251802 & -189.815481 & -189.811173 \\
\hline 36 & -367.6 & -367.687 & -268.279417 & -268.2 \\
\hline 37 & & & & \\
\hline 38 & -249.562562 & -249.556162 & -150.139301 & -150.134545 \\
\hline
\end{tabular}




\begin{tabular}{|l|l|l|l|l|}
\hline $\mathbf{3 9}$ & -570.222493 & -570.213467 & -470.794176 & -470.786129 \\
\hline $\mathbf{4 0}$ & -288.740382 & -288.733383 & -189.337602 & -189.331267 \\
\hline $\mathbf{4 1}$ & -480.456464 & -480.451180 & -380.999514 & -380.994885 \\
\hline $\mathbf{4 2}$ & -821.993528 & -821.986475 & -722.524257 & -722.517972 \\
\hline $\mathbf{4 3}$ & -1172.154113 & -1172.143361 & -1072.523299 & -1072.513645 \\
\hline $\mathbf{4 3}$ & -1172.117903 & -1172.106589 & & \\
\hline $\mathbf{4 4}$ & -770.741662 & -770.733996 & -671.273095 & -671.266347 \\
\hline $\mathbf{4 5}$ & -881.344364 & -881.333798 & -781.911282 & -781.901814 \\
\hline $\mathbf{4 6}$ & -999.102469 & -999.085998 & -899.675490 & -899.660541 \\
\hline $\mathbf{4 7}$ & -1155.996707 & -1155.974958 & -1056.590017 & -1056.568949 \\
\hline $\mathbf{4 8}$ & -600.303991 & -600.291644 & -500.844407 & -500.833134 \\
\hline $\mathbf{4 9}$ & -898.075222 & -898.067776 & -798.633319 & -798.627304 \\
\hline $\mathbf{5 0}$ & -899.279272 & -899.271263 & -799.836509 & -799.829387 \\
\hline $\mathbf{5 1}$ & -478.990405 & -478.981356 & -379.617345 & -379.610102 \\
\hline $\mathbf{5 2}$ & -310.727345 & -310.720536 & -211.351796 & -211.346060 \\
\hline
\end{tabular}

S-3 


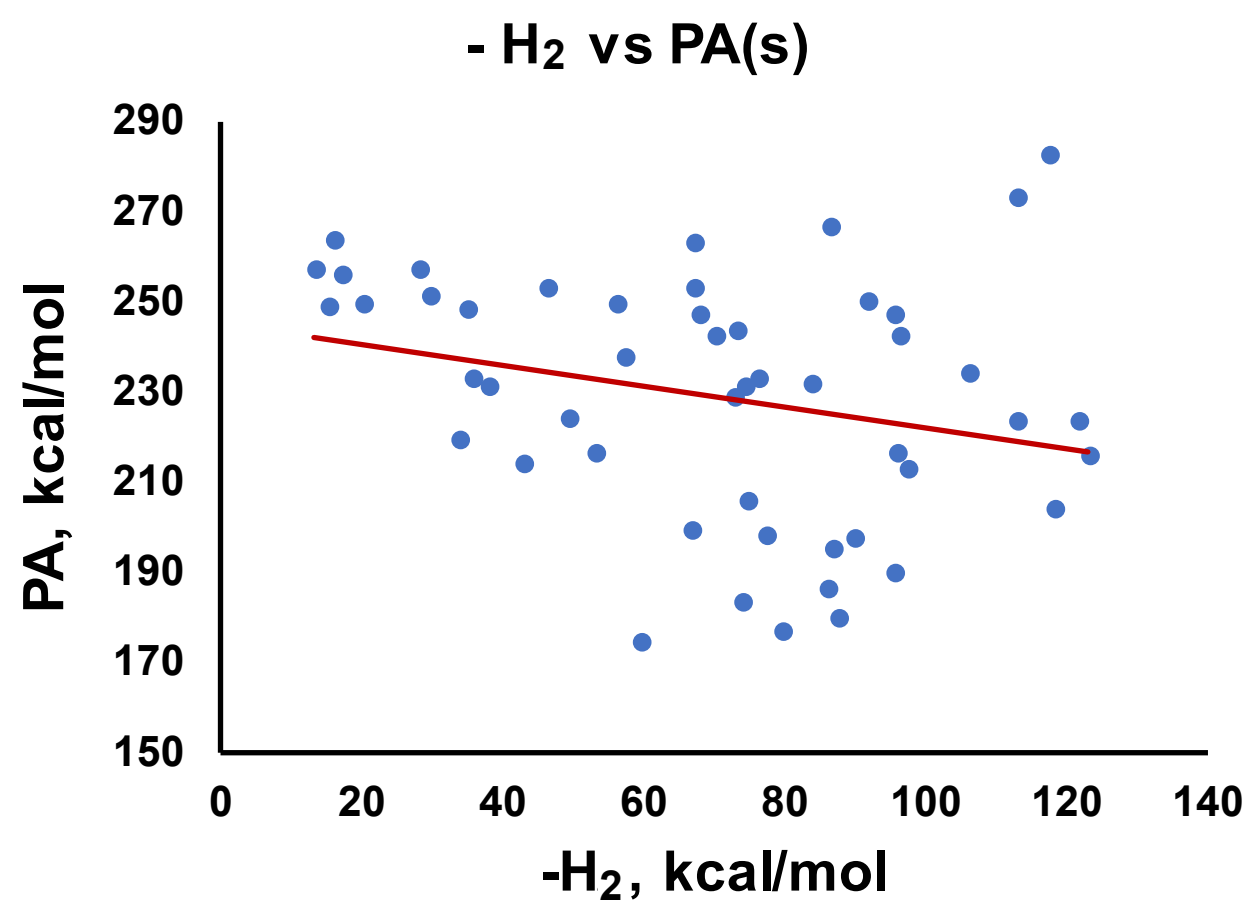

$-\mathrm{H}_{2}$ vs $\mathrm{FA}(\mathrm{S})$

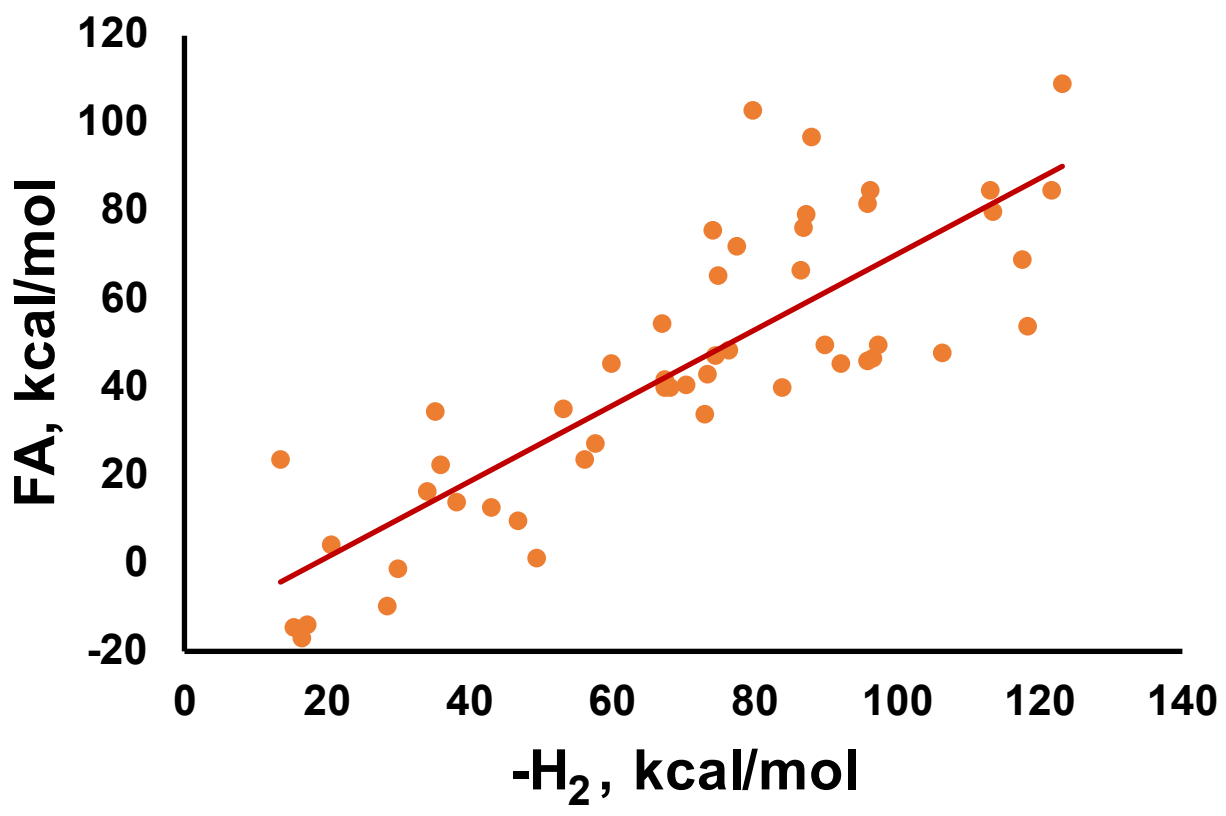

Figure S1. Hydride affinity (HA) and fluoride affinity vs. hydrogenation energy for carbenes. 


\section{S-T vs PA(S)}
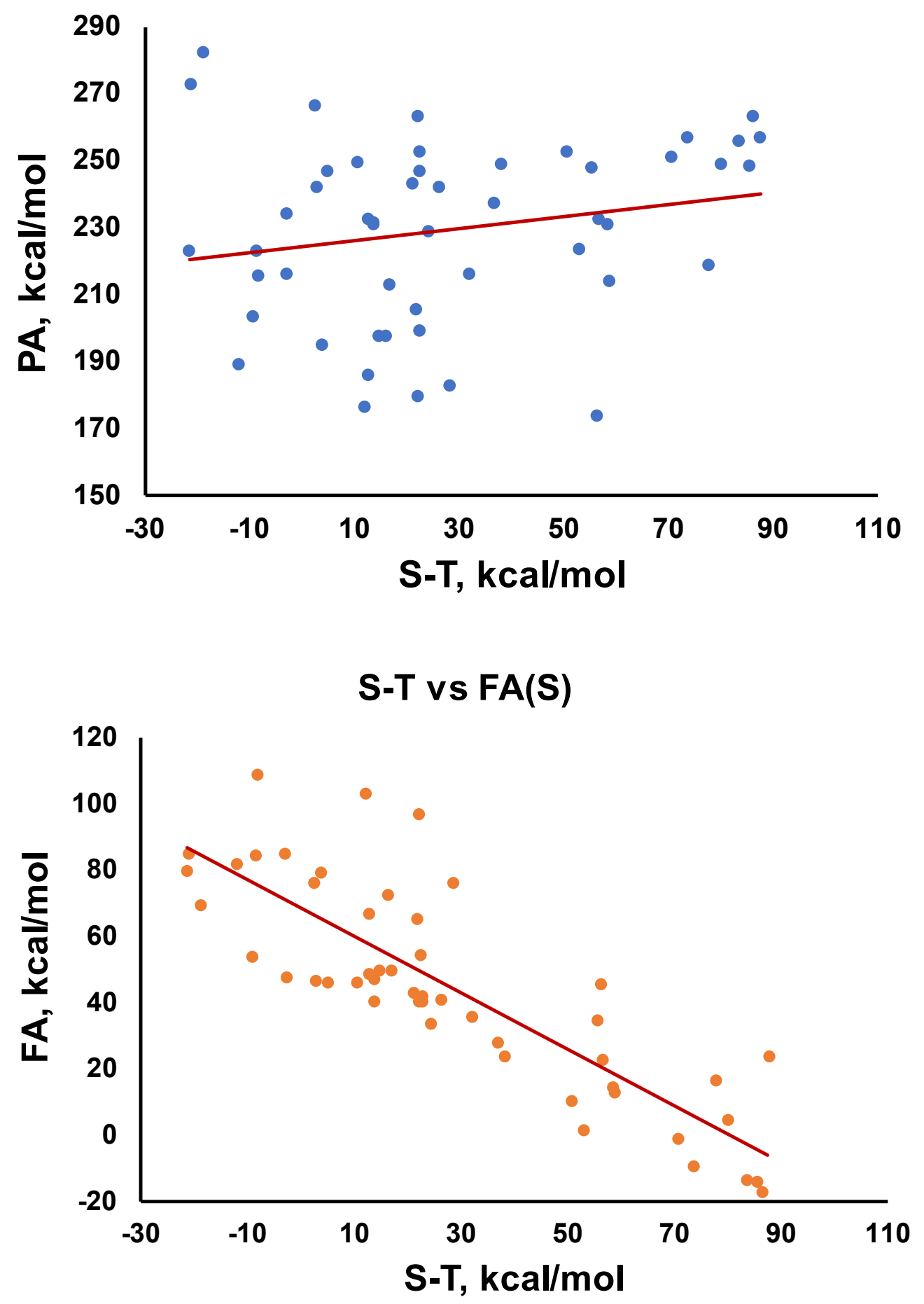

Figure S2. Hydride affinity (HA) and fluoride affinity vs. singlet-triplet splitting for carbenes. 


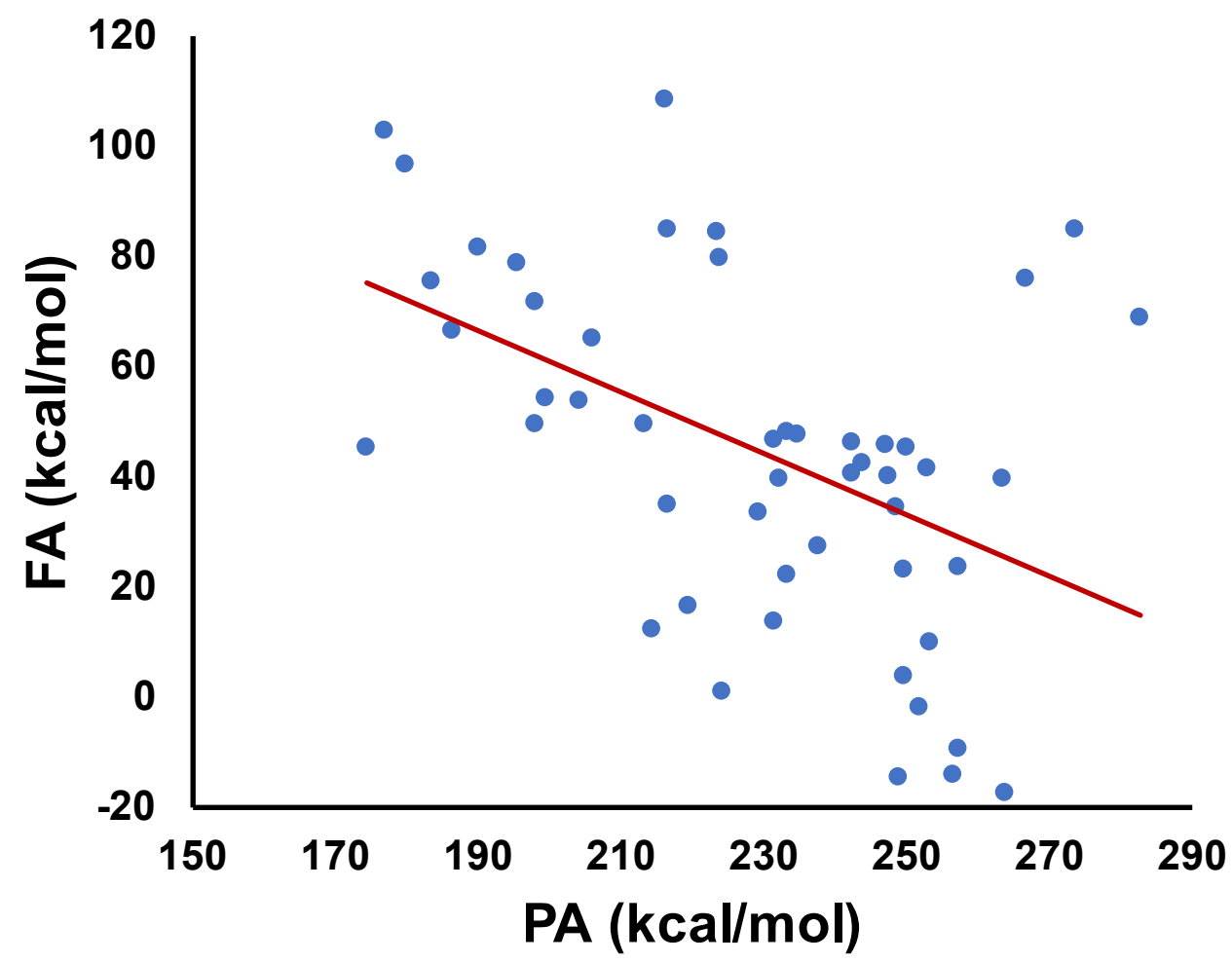

Figure S3. Hydride affinity (HA) vs. fluoride affinity for carbenes. 
Table S2. Optimized G3MP2 (MP2(FULL)/6-31G $(d)$ ) XYZ Coordinates in $\AA$.

$\begin{array}{cccc}1 \mathrm{~F} & & & \\ \mathrm{C} & -1.297130 & -0.512812 & 0.338535 \\ \mathrm{C} & -1.097173 & 0.814570 & 0.249107 \\ \mathrm{H} & -0.469333 & -1.691132 & -1.112018 \\ \mathrm{H} & -2.104121 & -1.035800 & 0.845509 \\ \mathrm{H} & -1.723700 & 1.611461 & 0.643973 \\ \mathrm{H} & 0.617349 & 1.815784 & -0.246647 \\ \mathrm{C} & 0.837110 & -0.190052 & -0.636438 \\ \mathrm{~N} & -0.193618 & -1.199032 & -0.263396 \\ \mathrm{~N} & 0.015393 & 1.069091 & -0.587120 \\ \mathrm{~F} & 1.585615 & -0.051218 & 0.679508 \\ & & & \\ \mathrm{~F} & & & \\ \mathrm{C} & 0.229560 & -1.036020 & 0.183505 \\ \mathrm{C} & 1.664579 & -0.709721 & -0.208134 \\ \mathrm{C} & 0.372385 & 1.271986 & 0.003654 \\ \mathrm{H} & -0.226382 & -1.824113 & -0.427409 \\ \mathrm{H} & 0.184343 & -1.359869 & 1.236507 \\ \mathrm{H} & 1.813434 & -0.869805 & -1.291515 \\ \mathrm{H} & 2.411258 & -1.306897 & 0.331711 \\ \mathrm{~N} & 1.697744 & 0.708938 & 0.164603 \\ \mathrm{~N} & -0.472214 & 0.256095 & 0.009822 \\ \mathrm{H} & 2.407747 & 1.259090 & -0.309762 \\ \mathrm{H} & -1.973026 & 0.190981 & -0.031891 \\ \mathrm{~F} & -2.977247 & -0.000232 & -0.066973 \\ & & & \\ 3 \mathrm{~F} & & & \\ \mathrm{C} & 0.782119 & -1.197410 & -0.000005 \\ \mathrm{C} & -0.508058 & -0.748665 & -0.000014 \\ \mathrm{H} & 1.184013 & -2.202196 & -0.000018 \\ \mathrm{H} & -1.558557 & -1.155476 & -0.000004 \\ \mathrm{C} & 0.894681 & 1.114175 & 0.000003 \\ \mathrm{~N} & 1.596983 & -0.073082 & 0.000000 \\ \mathrm{~N} & -0.387976 & 0.635786 & -0.000004 \\ \mathrm{C} & 3.037890 & -0.110549 & 0.000008 \\ \mathrm{H} & 3.423485 & -0.622777 & -0.889281 \\ \mathrm{H} & 3.380237 & 0.924307 & 0.000011 \\ \mathrm{H} & 3.423476 & -0.622781 & 0.889298 \\ \mathrm{C} & -1.590679 & 1.465794 & -0.000003 \\ \mathrm{H} & -1.590569 & 2.105516 & -0.887414 \\ \mathrm{H} & -2.441317 & 0.758061 & 0.000003 \\ \mathrm{H} & -1.590560 & 2.105523 & 0.887403 \\ \mathrm{~F} & -3.154331 & -0.929909 & 0.000011 \\ & & & \end{array}$




\begin{tabular}{|c|c|c|c|}
\hline \multicolumn{4}{|l|}{$+\mathrm{F}$} \\
\hline $\mathrm{C}$ & -0.579047 & -1.291638 & -0.423542 \\
\hline $\mathrm{C}$ & 0.939811 & -1.220535 & -0.434425 \\
\hline $\mathrm{C}$ & -0.019305 & 0.886309 & 0.262606 \\
\hline $\mathrm{H}$ & -0.996005 & -1.031192 & -1.410453 \\
\hline $\mathrm{H}$ & -0.957336 & -2.282948 & -0.130912 \\
\hline $\mathrm{H}$ & 1.386067 & -1.588513 & -1.372146 \\
\hline $\mathrm{H}$ & 1.337366 & -1.842913 & 0338 \\
\hline $\mathrm{N}$ & 1.197547 & 0.206947 & -0.205458 \\
\hline $\mathrm{N}$ & -0.861285 & $-0.2^{\prime}$ & 0.585859 \\
\hline $\mathrm{C}$ & 2.3 & 0.4 & \\
\hline $\mathrm{H}$ & 2.413717 & 1.501854 & 0.8 \\
\hline $\mathrm{H}$ & 2.26 & -0.088023 & \\
\hline $\mathrm{H}$ & 3.260295 & 0.08 & 0.170027 \\
\hline $\mathrm{C}$ & -2.2 & & \\
\hline $\mathrm{H}$ & -2.720080 & 0.351863 & -0.246247 \\
\hline $\mathrm{H}$ & -2.82 & -0.7 & 047 \\
\hline $\mathrm{H}$ & -2.34 & 0.9 & 1.3 \\
\hline $\mathrm{F}$ & -0.62 & 1.3 & -1.0 \\
\hline \multicolumn{4}{|l|}{$5 \mathrm{~F}$} \\
\hline $\mathrm{C}$ & -0.2 & -0.5 & \\
\hline $\mathrm{C}$ & -0.24 & -0.5 & 816 \\
\hline $\mathrm{C}$ & -0.16 & & \\
\hline $\mathrm{N}$ & -0.24 & 0.7 & 1.1 \\
\hline $\mathrm{N}$ & -0.24 & 0.7 & -1.1 \\
\hline $\mathrm{C}$ & -0.12 & -1.7 & -1.6 \\
\hline $\mathrm{H}$ & -0.92 & -1.6 & -2.3 \\
\hline $\mathrm{H}$ & 0.8 & $-1 .($ & -2.1 \\
\hline $\mathrm{H}$ & -0.1 & -2.6 & -1.1 \\
\hline $\mathrm{C}$ & -0.1 & -1.7 & 100 \\
\hline $\mathrm{H}$ & 0.8 & -1.6 & 2.1 \\
\hline $\mathrm{H}$ & -0.9 & -1.6 & 2.3 \\
\hline $\mathrm{H}$ & -0.1 & -2.6 & 9910 \\
\hline $\mathrm{H}$ & -1.0 & 630 & -1.7 \\
\hline $\mathrm{H}$ & -1.0 & 0.9 & 1.7 \\
\hline $\mathrm{F}$ & 1.296968 & 1.962396 & 0.000000 \\
\hline \multicolumn{4}{|l|}{$6 \mathrm{~F}$} \\
\hline $\mathrm{C}$ & 0.8 & 0.0 & 0.6 \\
\hline O & 0.8 & 0.0 & -0.6 \\
\hline $\mathrm{C}$ & -1.451146 & 0.12 & 0.000000 \\
\hline $\mathrm{N}$ & -0.513369 & 640 & 1.15670 \\
\hline $\mathrm{N}$ & -0.513369 & 0.039640 & -1.15670 \\
\hline $\mathrm{C}$ & -0.810122 & -1.056207 & 2.05824 \\
\hline$T$ & -0.580404 & -2.053963 & 1.63689 \\
\hline
\end{tabular}




$\begin{array}{crrr}\mathrm{H} & -0.251914 & -0.954766 & 3.000629 \\ \mathrm{H} & -1.879178 & -1.025594 & 2.275862 \\ \mathrm{C} & -0.810122 & -1.056207 & -2.058242 \\ \mathrm{H} & -0.251914 & -0.954766 & -3.000629 \\ \mathrm{H} & -0.580404 & -2.053963 & -1.636892 \\ \mathrm{H} & -1.879178 & -1.025594 & -2.275862 \\ \mathrm{C} & 1.961418 & 0.225524 & -1.624054 \\ \mathrm{H} & 2.072501 & -0.650475 & -2.276724 \\ \mathrm{H} & 1.814210 & 1.094355 & -2.278012 \\ \mathrm{H} & 2.907798 & 0.354754 & -1.087926 \\ \mathrm{C} & 1.961418 & 0.225524 & 1.624054 \\ \mathrm{H} & 1.814210 & 1.094355 & 2.278012 \\ \mathrm{H} & 2.072501 & -0.650475 & 2.276724 \\ \mathrm{H} & 2.907798 & 0.354754 & 1.087926 \\ \mathrm{~F} & -1.762015 & 1.577092 & 0.000000\end{array}$

$7 \mathrm{~F}$

$\begin{array}{llll}\text { C } & 0.061117 & 1.057841 & 0.000000\end{array}$

$\begin{array}{llll}\text { C } & 0.408656 & -1.117269 & 0.669488\end{array}$

$\begin{array}{llll}\text { C } & 0.408656 & -1.117269 & -0.669488\end{array}$

$\mathrm{H} \quad 0.430541 \quad-1.934224 \quad 1.380696$

$\mathrm{H} \quad 0.430541 \quad-1.934224 \quad-1.380696$

$\begin{array}{llll}\mathrm{O} & 0.408656 & 0.173325 & 1.143645\end{array}$

O $\quad 0.408656 \quad 0.173325 \quad-1.143645$

F $\quad-1.407794 \quad 0.906160 \quad 0.000000$

$8 \mathrm{~F}$

$\begin{array}{llll}\text { C } & -0.194748 & 1.156560 & 0.000000\end{array}$

$\begin{array}{llll}\text { C } & 0.348720 & -1.432232 & 0.673992\end{array}$

C $\quad 0.348720 \quad-1.432232 \quad-0.673992$

$\mathrm{H} \quad 0.364766-2.329832 \quad 1.286952$

H $\quad 0.364766 \quad-2.329832 \quad-1.286952$

S $\quad 0.348720 \quad 0.176109 \quad 1.412010$

S $\quad 0.348720 \quad 0.176109 \quad-1.412010$

$\begin{array}{llll}\text { F } & -1.656079 & 1.030177 & 0.000000\end{array}$

9F

$\begin{array}{llll}\text { C } & 0.395371 & 1.047284 & -0.101105\end{array}$

$\begin{array}{llll}\text { C } & -0.513863 & 0.000000 & 0.567986\end{array}$

C $\quad 0.395371 \quad-1.047284-0.101105$

$\begin{array}{llll}\text { C } & 1.517865 & 0.000000 & 0.019959\end{array}$

$\mathrm{H} \quad 0.176018 \quad 1.192961 \quad-1.180212$

H $\quad 0.539626 \quad 2.041876 \quad 0.356472$

H $\quad 0.176018-1.192961 \quad-1.180212$

$\mathrm{H} \quad 0.539626 \quad-2.041876 \quad 0.356471$

$\begin{array}{llll}\mathrm{H} & 2.324951 & 0.000000 & -0.724763\end{array}$ 


$$
\begin{array}{crcc}
\mathrm{H} & 1.954374 & 0.000000 & 1.022566 \\
\mathrm{~F} & -1.831009 & 0.000000 & -0.107192 \\
& & & \\
10 \mathrm{~F} & & & \\
\mathrm{C} & -2.024471 & 0.164579 & -0.000003 \\
\mathrm{C} & 1.618429 & -0.722154 & -0.000001 \\
\mathrm{C} & 0.440923 & -0.065633 & 0.000000 \\
\mathrm{C} & -0.904641 & -0.617798 & 0.000003 \\
\mathrm{H} & 2.561660 & -0.189152 & 0.000002 \\
\mathrm{H} & 1.633456 & -1.804892 & -0.000008 \\
\mathrm{H} & -2.890310 & -0.550275 & -0.000003 \\
\mathrm{H} & -0.852411 & -1.724787 & 0.000008 \\
\mathrm{~F} & 0.529574 & 1.301682 & 0.000001 \\
& & & \\
11 \mathrm{~F} & & & \\
\mathrm{C} & -0.138411 & -0.823254 & 0.750371 \\
\mathrm{C} & 0.720750 & 0.205498 & 0.000000 \\
\mathrm{C} & -0.138411 & -0.823254 & -0.750371 \\
\mathrm{H} & 0.358537 & -1.626183 & 1.299371 \\
\mathrm{H} & -1.067471 & -0.493071 & 1.216150 \\
\mathrm{H} & -1.067471 & -0.493071 & -1.216150 \\
\mathrm{H} & 0.358537 & -1.626183 & -1.299371 \\
\mathrm{~F} & -0.138411 & 1.431619 & 0.000000 \\
& & & \\
12 \mathrm{~F} & & & \\
\mathrm{C} & 0.139650 & 0.001771 & 0.000000 \\
\mathrm{C} & 0.139650 & -0.701253 & 1.191737 \\
\mathrm{C} & 0.139650 & -0.701253 & -1.191737 \\
\mathrm{H} & 0.937510 & -1.464640 & -1.098236 \\
\mathrm{H} & 0.937510 & -1.464640 & 1.098236 \\
\mathrm{~F} & -0.487635 & 1.259299 & 0.000000 \\
& & & \\
13 \mathrm{~F} & & & \\
\mathrm{C} & -0.082222 & 0.779683 & 0.000000 \\
\mathrm{H} & 0.616661 & 0.976603 & 0.862172 \\
\mathrm{H} & 0.616661 & 0.976603 & -0.862172 \\
\mathrm{~F} & -0.082222 & -0.736811 & 0.000000 \\
& & & \\
14 \mathrm{~F} & & & \\
\mathrm{C} & 0.043984 & 0.670866 & 0.000000 \\
\mathrm{H} & -1.055624 & 0.938000 & 0.000000 \\
\mathrm{~F} & 0.043984 & -0.275733 & 1.134042 \\
\mathrm{~F} & 0.043984 & -0.275733 & -1.134042 \\
& & & \\
15 \mathrm{~F} & & & \\
\mathrm{C} & 0.000000 & 0.000000 & 0.563674 \\
& & &
\end{array}
$$

$\begin{array}{lrrr}\mathrm{C} & -0.082222 & 0.779683 & 0.000000 \\ \mathrm{H} & 0.616661 & 0.976603 & 0.862172 \\ \mathrm{H} & 0.616661 & 0.976603 & -0.862172 \\ \mathrm{~F} & -0.082222 & -0.736811 & 0.000000\end{array}$




$\begin{array}{cccc}\text { F } & 0.000000 & 1.270070 & -0.125261 \\ \text { F } & 1.099913 & -0.635035 & -0.125261 \\ \text { F } & -1.099913 & -0.635035 & -0.125261 \\ & & & \\ 16 \mathrm{~F} & & & \\ \mathrm{C} & 1.145998 & -0.215270 & 0.018348 \\ \mathrm{C} & -0.071390 & 0.678702 & -0.205873 \\ \mathrm{H} & 1.277329 & -0.928724 & -0.811330 \\ \mathrm{H} & 2.056756 & 0.405733 & 0.041807 \\ \mathrm{H} & 1.153602 & -0.833333 & 0.945590 \\ \mathrm{H} & -0.131889 & 1.286273 & 0.734149 \\ \mathrm{~F} & -1.200383 & -0.301171 & 0.023882 \\ & & & \\ 17 \mathrm{~F} & & & \\ \mathrm{C} & -0.388120 & -0.497920 & 1.218808 \\ \mathrm{C} & -0.388120 & 0.403741 & 0.000000 \\ \mathrm{H} & -0.212198 & 0.075678 & 2.140146 \\ \mathrm{H} & -1.367464 & -0.988489 & 1.328917 \\ \mathrm{H} & 0.382601 & -1.309049 & 1.197445 \\ \mathrm{C} & -0.388120 & -0.497920 & -1.218808 \\ \mathrm{H} & 0.382601 & -1.309049 & -1.197445 \\ \mathrm{H} & -1.367464 & -0.988489 & -1.328917 \\ \mathrm{H} & -0.212198 & 0.075678 & -2.140146 \\ \mathrm{~F} & 1.042254 & 0.888480 & 0.000000\end{array}$

$18 \mathrm{~F}$

$\begin{array}{cccc}\mathrm{C} & -2.052082 & -0.460992 & 0.621461 \\ \mathrm{C} & -1.371095 & -0.160496 & -0.728590 \\ \mathrm{H} & -3.138937 & -0.604754 & 0.531714 \\ \mathrm{H} & -1.638284 & -1.359486 & 1.091083 \\ \mathrm{H} & -1.863661 & 0.379968 & 1.292960 \\ \mathrm{C} & 0.088149 & 0.283216 & -0.705269 \\ \mathrm{H} & -1.445659 & -1.047280 & -1.379183 \\ \mathrm{H} & -1.967348 & 0.621964 & -1.225419 \\ \mathrm{C} & 0.933610 & -0.722903 & 0.053107 \\ \mathrm{H} & 0.605944 & -0.930851 & 1.097923 \\ \mathrm{H} & 0.884726 & -1.686036 & -0.482153 \\ \mathrm{C} & 2.388997 & -0.263036 & 0.123350 \\ \mathrm{H} & 2.423690 & 0.697413 & 0.643913 \\ \mathrm{H} & 3.045303 & -0.971772 & 0.650864 \\ \mathrm{H} & 2.769640 & -0.102364 & -0.889902 \\ \mathrm{~F} & 0.044346 & 1.438719 & 0.275983\end{array}$

$19 \mathrm{~F}$

$\begin{array}{llll}\text { C } & 0.319638 & -0.069014 & -0.040301\end{array}$

$\begin{array}{llll}\text { C } & -0.890629 & -0.836224 & -0.291026\end{array}$ 


$\begin{array}{cccc}\text { F } & 0.502492 & 0.586544 & 1.200858 \\ \text { F } & 0.525468 & 0.968117 & -0.924842 \\ \text { F } & 1.434011 & -0.873550 & -0.131969 \\ \text { H } & -0.927336 & -1.593927 & 0.510333 \\ \text { F } & -1.978273 & 0.099483 & 0.020133 \\ & & & \\ \text { 20F } & & & \\ \text { C } & -1.221977 & -0.111657 & -0.083888 \\ \text { C } & -0.000001 & 0.489379 & -0.615356 \\ \text { F } & 1.337488 & -1.428690 & -0.427101 \\ \text { F } & 2.346446 & 0.516277 & -0.552916 \\ \text { F } & 1.415980 & -0.098875 & 1.296559 \\ \text { F } & -2.346456 & 0.516225 & -0.552963 \\ \text { F } & -1.416004 & -0.098815 & 1.296554 \\ \text { F } & -1.337450 & -1.428709 & -0.427045 \\ \text { C } & 1.221977 & -0.111654 & -0.083889 \\ \text { F } & -0.000003 & 1.845208 & -0.111000 \\ & & & \\ 21 F & & & \\ \text { C } & -1.594508 & -0.303116 & 0.000007 \\ \text { SI } & 0.009330 & 0.386517 & 0.000023 \\ \text { H } & 0.271573 & 1.240421 & 1.220090 \\ \text { H } & 0.271476 & 1.240530 & -1.219987 \\ \text { H } & -2.009323 & -0.739081 & 0.910037 \\ \text { H } & -2.009435 & -0.738675 & -0.910174 \\ \text { F } & 1.434682 & -0.510637 & -0.000036 \\ & & & \\ 321 F & & & \\ \text { C } & -1.371068 & -0.767813 & 0.000000 \\ \text { SI } & -0.004437 & 0.536570 & 0.000001 \\ \text { H } & 0.527740 & 1.140828 & 1.289758 \\ \text { H } & 0.527772 & 1.140839 & -1.289740 \\ \text { H } & -1.203512 & 1.597614 & -0.000014 \\ \text { H } & -2.462725 & -0.721777 & -0.000010 \\ \text { F } & 1.211027 & -0.673622 & -0.000001\end{array}$

$\begin{array}{crrr}22 \mathrm{~F} & & & \\ \mathrm{C} & -0.000061 & 0.291916 & 0.000000 \\ \mathrm{H} & -1.158617 & -0.161024 & 2.557301 \\ \mathrm{H} & -0.000499 & -1.954033 & 1.467497 \\ \mathrm{H} & 1.158843 & -0.161728 & 2.557109 \\ \mathrm{SI} & -0.000061 & -0.461665 & 1.623550 \\ \mathrm{SI} & -0.000061 & -0.461665 & -1.623550 \\ \mathrm{H} & 1.158843 & -0.161728 & -2.557109 \\ \mathrm{H} & -0.000499 & -1.954033 & -1.467497 \\ \mathrm{H} & -1.158617 & -0.161024 & -2.557301\end{array}$




$\begin{array}{cccc}\text { F } & 0.000290 & 1.747632 & 0.000000 \\ & & & \\ 23 \mathrm{~F} & & & \\ \mathrm{C} & -1.330011 & -0.907283 & 0.044448 \\ \mathrm{SI} & 0.263638 & -0.070648 & 0.008688 \\ \mathrm{C} & 1.644295 & -1.362570 & -0.277054 \\ \mathrm{H} & 1.437256 & -1.970372 & -1.161967 \\ \mathrm{H} & 2.618109 & -0.877478 & -0.413156 \\ \mathrm{H} & 1.734976 & -2.040727 & 0.579134 \\ \mathrm{C} & 0.295116 & 1.149975 & -1.457984 \\ \mathrm{H} & -0.574173 & 1.812917 & -1.389128 \\ \mathrm{H} & 1.197218 & 1.773320 & -1.488072 \\ \mathrm{H} & 0.214913 & 0.608156 & -2.404926 \\ \mathrm{C} & 1.013972 & 1.012792 & 1.431243 \\ \mathrm{H} & 1.173829 & 0.409315 & 2.332797 \\ \mathrm{H} & 1.976575 & 1.469658 & 1.160599 \\ \mathrm{H} & 0.329228 & 1.825167 & 1.702690 \\ \mathrm{H} & -1.489372 & -1.707982 & 0.781114 \\ \mathrm{~F} & -2.449969 & 0.036625 & 0.192818 \\ & & & \\ \text { 24F } & & & \\ \mathrm{C} & -0.149111 & -0.813000 & 0.000230 \\ \mathrm{SI} & 1.609173 & -0.074886 & 0.000024 \\ \mathrm{C} & 2.401117 & 0.489680 & -1.657997 \\ \mathrm{H} & 2.732288 & 1.530602 & -1.593562 \\ \mathrm{H} & 3.240203 & -0.133378 & -1.983128 \\ \mathrm{H} & 1.635004 & 0.457302 & -2.442065 \\ \mathrm{C} & 2.401149 & 0.490576 & 1.657723 \\ \mathrm{H} & 1.635023 & 0.458744 & 2.441800 \\ \mathrm{H} & 3.240176 & -0.132360 & 1.983237 \\ \mathrm{H} & -2.159414 & 0.913324 & 1.531208 \\ \mathrm{H} & 2.732414 & 1.531431 & 1.592671 \\ \mathrm{C} & 2.461619 & -1.859121 & 0.000485 \\ \mathrm{H} & 3.560469 & -1.794178 & 0.000467 \\ \mathrm{H} & 2.175776 & -2.448544 & 0.883335 \\ \mathrm{H} & 2.175773 & -2.449003 & -0.882056 \\ \mathrm{SI} & -1.864667 & -0.157768 & 0.000044 \\ \mathrm{C} & -3.167747 & -1.543107 & 0.000370 \\ \mathrm{H} & -4.184591 & -1.134030 & 0.000254 \\ \mathrm{H} & -3.063168 & -2.181503 & -0.882657 \\ \mathrm{H} & -2.159366 & 0.912560 & -1.531664 \\ \mathrm{H} & -139403 & 1.402907 & -1.519676 \\ \mathrm{H} & & & \\ \mathrm{H} & -1.674357 & 1.548878\end{array}$




$$
\begin{array}{cccc}
\mathrm{H} & -3.139455 & 1.403658 & 1.518948 \\
\mathrm{H} & -2.083751 & 0.324655 & 2.450992 \\
\mathrm{~F} & 0.687650 & 1.487231 & -0.000377 \\
& & & \\
25 \mathrm{~F} & & & \\
\mathrm{C} & 0.650644 & 0.302682 & -0.000007 \\
\mathrm{O} & 1.873724 & -0.073685 & 0.000022 \\
\mathrm{C} & -0.489899 & -0.453168 & -0.000072 \\
\mathrm{H} & 0.406372 & 1.401077 & -0.000049 \\
\mathrm{H} & -0.569420 & -1.534206 & 0.000096 \\
\mathrm{~F} & -1.754579 & 0.180614 & 0.000028 \\
& & & \\
{ }^{3} 25 \mathrm{~F} & & & \\
\mathrm{C} & 0.765059 & -0.380131 & 0.239973 \\
\mathrm{O} & 1.710770 & 0.423945 & -0.151147 \\
\mathrm{C} & -0.556556 & -0.449008 & -0.333919 \\
\mathrm{H} & 0.838734 & -0.873243 & 1.242431 \\
\mathrm{H} & -1.057414 & -1.395992 & -0.558239 \\
\mathrm{~F} & -1.635388 & 0.428057 & 0.120961
\end{array}
$$

$\begin{array}{crrr}26 \mathrm{~F} & & & \\ \mathrm{C} & -0.920918 & -0.537297 & -0.000014 \\ \mathrm{O} & -2.011767 & 0.137577 & -0.000053 \\ \mathrm{H} & -0.959806 & -1.659019 & -0.000055 \\ \mathrm{C} & 0.367894 & -0.071362 & 0.000345 \\ \mathrm{C} & 0.799899 & 1.342130 & -0.000044 \\ \mathrm{H} & 1.397646 & 1.626150 & 0.882786 \\ \mathrm{H} & 1.396253 & 1.626153 & -0.883831 \\ \mathrm{H} & -0.121503 & 1.932546 & 0.000658 \\ \mathrm{~F} & 1.433365 & -1.003030 & -0.000095\end{array}$

$27 \mathrm{~F}$
$\begin{array}{llll}\text { C } & -0.242271 & 0.781571 & 0.000000\end{array}$
$\begin{array}{llll}\text { CL } & -0.242271 & -0.452845 & 1.530584\end{array}$
$\begin{array}{llll}\text { CL } & -0.242271 & -0.452845 & -1.530584\end{array}$
F $\quad 1.076759 \quad 1.189699 \quad 0.000000$

$$
\begin{array}{cccc}
28 \mathrm{~F} & & & \\
\mathrm{C} & 0.052845 & 0.419016 & 0.561599 \\
\mathrm{CL} & -1.381200 & -0.617741 & -0.064416 \\
\mathrm{C} & 1.246849 & -0.241454 & 0.059791 \\
\mathrm{~N} & 2.303766 & -0.750719 & -0.137679 \\
\mathrm{~F} & -0.049347 & 1.632362 & -0.185503
\end{array}
$$

29F

$\begin{array}{llll}\text { C } & 0.116941 & 0.436072 & 0.607860\end{array}$ 


\begin{tabular}{|c|c|c|c|}
\hline $\mathrm{CL}$ & -1.393211 & -0.549959 & -0.066548 \\
\hline $\mathrm{N}$ & 1.229788 & -0.310916 & 0.058930 \\
\hline $\mathrm{C}$ & 2.242052 & -0.901090 & -0.172145 \\
\hline $\mathrm{F}$ & 0.102456 & 1.590648 & -0.210610 \\
\hline \multicolumn{4}{|l|}{$30 \mathrm{~F}$} \\
\hline $\mathrm{C}$ & -0.659697 & 0.103523 & 0.000000 \\
\hline $\mathrm{C}$ & 0.699038 & 0.686477 & 0.000000 \\
\hline $\mathrm{N}$ & 1.662644 & 1.382626 & 0.000000 \\
\hline $\mathrm{F}$ & -0.659697 & -0.801021 & 1.121662 \\
\hline $\mathrm{F}$ & -0.659697 & -0.801021 & -1.121662 \\
\hline \multicolumn{4}{|l|}{$31 \mathrm{~F}$} \\
\hline $\mathrm{C}$ & -0.668516 & 0.193800 & 0.000000 \\
\hline $\mathrm{N}$ & 0.755510 & 0.632916 & 0.000000 \\
\hline $\mathrm{C}$ & 1.792637 & 1.222148 & 0.000000 \\
\hline $\mathrm{F}$ & -0.668516 & -0.718117 & 1.105069 \\
\hline $\mathrm{F}$ & -0.668516 & -0.718117 & -1.105069 \\
\hline \multicolumn{4}{|l|}{$32 \mathrm{~F}$} \\
\hline $\mathrm{C}$ & 0.000000 & 0.286726 & 0.217712 \\
\hline $\mathrm{C}$ & -1.223705 & -0.356284 & 0.024763 \\
\hline $\mathrm{C}$ & 1.223705 & -0.356284 & 0.024763 \\
\hline $\mathrm{N}$ & -2.285093 & -0.892763 & -0.067193 \\
\hline $\mathrm{N}$ & 2.285093 & -0.892763 & -0.067193 \\
\hline $\mathrm{F}$ & 0.000000 & 1.672636 & -0.073636 \\
\hline \multicolumn{4}{|l|}{$33 \mathrm{~F}$} \\
\hline $\mathrm{C}$ & -0.369114 & 0.513243 & 0.000000 \\
\hline $\mathrm{N}$ & -0.174525 & -0.321275 & 1.141495 \\
\hline $\mathrm{C}$ & -0.174525 & -0.932797 & 2.168216 \\
\hline $\mathrm{N}$ & -0.174525 & -0.321275 & -1.141495 \\
\hline $\mathrm{C}$ & -0.174525 & -0.932797 & -2.168216 \\
\hline $\mathrm{F}$ & 0.750260 & 1.401329 & 0.000000 \\
\hline \multicolumn{4}{|l|}{$34 \mathrm{~F}$} \\
\hline $\mathrm{C}$ & -0.988288 & -0.049932 & 0.044640 \\
\hline $\mathrm{N}$ & -2.170961 & -0.082831 & -0.088747 \\
\hline $\mathrm{C}$ & 0.401074 & -0.008777 & 0.470815 \\
\hline $\mathrm{O}$ & 1.078255 & -1.085715 & -0.223147 \\
\hline $\mathrm{H}$ & 1.733291 & -1.371190 & 0.429549 \\
\hline $\mathrm{F}$ & 0.928964 & 1.220998 & -0.123986 \\
\hline \multicolumn{4}{|l|}{$35 \mathrm{~F}$} \\
\hline $\mathrm{C}$ & -0.382655 & 0.362116 & 0.000000 \\
\hline $\mathrm{O}$ & -0.382655 & -0.589307 & 1.105271 \\
\hline
\end{tabular}




$\begin{array}{cccc}\mathrm{H} & -0.588644 & 0.001436 & 1.843916 \\ \mathrm{O} & -0.382655 & -0.589307 & -1.105271 \\ \mathrm{H} & -0.588644 & 0.001436 & -1.843916 \\ \mathrm{~F} & 1.066189 & 0.805928 & 0.000000 \\ & & & \\ 36 \mathrm{~F} & & & \\ \mathrm{C} & -0.276690 & 0.062500 & 2.299899 \\ \mathrm{H} & -0.586534 & -0.622531 & 3.101104 \\ \mathrm{H} & 0.718325 & 0.461673 & 2.549148 \\ \mathrm{H} & -0.971661 & 0.914058 & 2.252695 \\ \mathrm{C} & -0.244951 & 0.304292 & 0.000000 \\ \mathrm{C} & -0.276690 & 0.062500 & -2.299899 \\ \mathrm{H} & -0.586534 & -0.622531 & -3.101104 \\ \mathrm{H} & -0.971661 & 0.914058 & -2.252695 \\ \mathrm{H} & 0.718325 & 0.461673 & -2.549148 \\ \mathrm{O} & -0.276690 & -0.648499 & 1.094738 \\ \mathrm{O} & -0.276690 & -0.648499 & -1.094738 \\ \mathrm{~F} & 1.210752 & 0.699316 & 0.000000 \\ & & & \\ 37 \mathrm{~F} & & & \\ \mathrm{C} & 0.982518 & 0.005750 & 0.062776 \\ \mathrm{~N} & 2.165086 & 0.089390 & -0.090380 \\ \mathrm{C} & -0.396087 & -0.025821 & 0.431288 \\ \mathrm{~N} & -1.162268 & 1.072853 & -0.118307 \\ \mathrm{H} & -0.762238 & 1.499850 & -0.959147 \\ \mathrm{H} & -1.264981 & 1.805240 & 0.575721 \\ \mathrm{~F} & -0.945678 & -1.257819 & -0.124461\end{array}$

38F.A

$\begin{array}{lrrl}\mathrm{N} & -0.553447 & -0.489614 & 1.148698 \\ \mathrm{H} & -0.312388 & 0.086139 & 1.948734 \\ \mathrm{H} & 0.166843 & -1.229115 & 1.089270 \\ \mathrm{C} & -0.553447 & 0.351515 & 0.000000 \\ \mathrm{~N} & -0.553447 & -0.489614 & -1.148698 \\ \mathrm{H} & -0.312388 & 0.086139 & -1.948734 \\ \mathrm{H} & 0.166843 & -1.229115 & -1.089270 \\ \mathrm{~F} & 1.262225 & 0.781273 & 0.000000\end{array}$

38F.B

$\begin{array}{rrrr}\mathrm{N} & -0.000718 & 0.548271 & 1.105955 \\ \mathrm{H} & -0.000460 & 0.997120 & 2.007067 \\ \mathrm{H} & 0.000061 & -0.496976 & 0.991280 \\ \mathrm{C} & -0.000718 & 1.345227 & 0.000000 \\ \mathrm{~N} & -0.000718 & 0.548271 & -1.105955 \\ \mathrm{H} & -0.000460 & 0.997120 & -2.007067 \\ \mathrm{H} & 0.000061 & -0.496976 & -0.991280\end{array}$




\begin{tabular}{|c|c|c|c|}
\hline $\mathrm{F}$ & 0.001684 & -1.860826 & 0.00 \\
\hline \multicolumn{4}{|c|}{$39 F$} \\
\hline $\mathrm{C}$ & -1.068785 & -0.077969 & -0.008493 \\
\hline $\mathrm{C}$ & 0.264936 & -0.648620 & -0.364945 \\
\hline $\mathrm{C}$ & 1.410731 & -0.122454 & 0.483111 \\
\hline $\mathrm{H}$ & 0.174838 & -1.729795 & -0.215181 \\
\hline $\mathrm{H}$ & 0.388997 & -0.466679 & -1.455324 \\
\hline $\mathrm{N}$ & 2.571334 & -0.833313 & -0.028401 \\
\hline $\mathrm{H}$ & 2.732845 & -0.565772 & -1.016917 \\
\hline $\mathrm{H}$ & 3.388462 & -0.482978 & 0.469425 \\
\hline $\mathrm{F}$ & -1.172573 & 1.258964 & -0.186358 \\
\hline $\mathrm{F}$ & -1.442743 & -0.330129 & 1.271127 \\
\hline $\mathrm{F}$ & -2.073051 & -0.62 & -0.787445 \\
\hline $\mathrm{F}$ & 1.541058 & 1.26 & -0.101905 \\
\hline \multicolumn{4}{|c|}{$40 \mathrm{~F}$} \\
\hline $\mathrm{C}$ & -0.649822 & -0.7 & 417 \\
\hline $\mathrm{C}$ & 0.474227 & -0.065099 & 7693 \\
\hline $\mathrm{H}$ & -0.50 & 3905 & 7723 \\
\hline $\mathrm{H}$ & -0.71 & 1044 & -0.066986 \\
\hline $\mathrm{N}$ & 1.6 & -0.6 & -0.018981 \\
\hline $\mathrm{H}$ & 1.774260 & 888 & 0.995944 \\
\hline $\mathrm{H}$ & & & \\
\hline $\mathrm{N}$ & -1.96 & 622 & -0.0 \\
\hline $\mathrm{H}$ & -1.765084 & 623 & 5600 \\
\hline $\mathrm{H}$ & -1.9 & -0.0 & 1398 \\
\hline $\mathrm{F}$ & 0.404046 & 1.3 & 0.136054 \\
\hline \multicolumn{4}{|c|}{$41 \mathrm{~F}$} \\
\hline $\mathrm{P}$ & & -0 . & 992 \\
\hline $\mathrm{H}$ & -1.70 & 496 & 4206 \\
\hline $\mathrm{H}$ & & & -0 . \\
\hline $\mathrm{C}$ & 0.55 & 0.6 & -0.096522 \\
\hline $\mathrm{H}$ & 0.7 & 067 & 505 \\
\hline $\mathrm{F}$ & 1.63 & -0.3 & 0.006338 \\
\hline \multicolumn{4}{|c|}{$42 \mathrm{~F}$} \\
\hline $\mathrm{P}$ & 1.58 & -0.3 & -0.0 \\
\hline $\mathrm{C}$ & 0.000000 & 0.317388 & 0.301609 \\
\hline $\mathrm{P}$ & -1.581004 & -0.393963 & -0.014528 \\
\hline $\mathrm{H}$ & & & -1.195706 \\
\hline $\mathrm{H}$ & -1.537518 & -1.531157 & 0.844925 \\
\hline $\mathrm{H}$ & 1.537516 & -1.531163 & 0.844919 \\
\hline $\mathrm{H}$ & 1.526977 & -1.253635 & -1.195710 \\
\hline $\mathrm{F}$ & 0.000001 & 1.720464 & -0.074696 \\
\hline
\end{tabular}




$$
\begin{array}{crrc}
\multicolumn{4}{l}{\text { 43F.A }} \\
\text { C } & 1.476646 & 0.001385 & 0.041482 \\
\text { C } & 0.107855 & 0.584383 & -0.233039 \\
\text { S } & -1.321651 & -0.263397 & -0.439494 \\
\text { F } & 1.987502 & -0.742012 & -0.980878 \\
F & 1.585233 & -0.765899 & 1.168264 \\
F & 2.365498 & 1.032654 & 0.217803 \\
\text { F } & 0.057691 & 1.896881 & 0.171345 \\
\text { F } & -0.340854 & -1.792043 & -0.474719 \\
F & -2.257987 & 1.255219 & -0.338118 \\
\text { F } & -2.103815 & -0.807051 & 1.145330
\end{array}
$$

$$
\begin{array}{crrr}
\multicolumn{4}{l}{\text { 43F.B }} \\
\text { C } & 1.604177 & -0.224315 & -0.029337 \\
\text { C } & 0.382383 & 0.612351 & -0.149823 \\
\text { S } & -1.271078 & 0.389195 & -0.048023 \\
\text { F } & -3.234869 & 0.171542 & -0.389942 \\
F & -1.516661 & -0.500849 & 1.440601 \\
\text { F } & -1.210274 & -1.079683 & -0.828922 \\
\text { F } & 2.346450 & 0.069553 & 1.097274 \\
\text { F } & 1.337429 & -1.547688 & 0.013012 \\
\text { F } & 2.486428 & -0.027820 & -1.074957 \\
\text { F } & 0.726818 & 1.964353 & -0.052252
\end{array}
$$




\begin{tabular}{|c|c|c|c|}
\hline $\mathrm{N}$ & -1.497926 & 213 & \\
\hline $\mathrm{H}$ & -1.086334 & 0.556344 & -2.0 \\
\hline-1 & -2.510379 & 0.844544 & -1.301887 \\
\hline $\mathrm{F}$ & 266 & -1.770551 & -0074966 \\
\hline \multicolumn{4}{|l|}{$46 \mathrm{~F}$} \\
\hline D & 1.695740 & 0.684030 & 0.000562 \\
\hline & 0.290 & 24 & 0.000000 \\
\hline N1 & -1.461244 & 0.08 & 0.000095 \\
\hline - & 2.768 & 0.04 & -1.2 \\
\hline $\mathrm{H}$ & 2.723960 & -0.9 & -1.2 \\
\hline Г & 2.292942 & 0.273002 & 359 \\
\hline $\mathrm{N}$ & 2.768 & & \\
\hline $\mathrm{H}$ & 2.293 & 0.26 & 2.1 \\
\hline $\mathrm{H}$ & 2.72 & -0.9 & \\
\hline $\mathrm{C}$ & -1.730690 & 1.96 & 0.0 \\
\hline $\mathrm{H}$ & -1.2 & & -0.8 \\
\hline $\mathrm{H}$ & -2.8 & 2.1 & 87 \\
\hline $\mathrm{H}$ & -1.2 & & \\
\hline $\mathrm{C}$ & -2.4 & -0.6 & -1 \\
\hline $\mathrm{H}$ & -2.2 & -1.6 & -1 . \\
\hline $\mathrm{H}$ & -3.5 & -0.4 & \\
\hline $\mathrm{H}$ & -2.07 & -0.150559 & 589 \\
\hline $\mathrm{C}$ & -2.42 & -0.60 & \\
\hline $\mathrm{H}$ & -3.5 & -0.4 & 36 \\
\hline $\mathrm{H}$ & & -1.6 & \\
\hline $\mathrm{H}$ & $-2.0^{\prime}$ & -0.1 & 42 \\
\hline $\mathrm{F}$ & 0.56 & -1.7 & -0. \\
\hline \multicolumn{4}{|l|}{$47 \mathrm{~F}$} \\
\hline D & -1.1 & 0.0 & \\
\hline 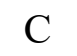 & 0.5 & 0.2 & \\
\hline S & 1.9 & 0.0 & -0.2 \\
\hline $\mathrm{N}$ & -2.03 & 1.16 & -0.2 \\
\hline $\mathrm{N}$ & & & \\
\hline $\mathrm{C}$ & 1.47 & -0.6 & -1 \\
\hline $\mathrm{H}$ & 1.20 & 0.2 & -2 \\
\hline $\mathrm{H}$ & 2.32 & -1.1 & -2 \\
\hline $\mathrm{H}$ & 0.62 & -1.2 & -1 . \\
\hline $\mathrm{C}$ & 2.98 & 1.594011 & -0.4 \\
\hline $\mathrm{H}$ & 3.291405 & 1.984268 & \\
\hline $\mathrm{H}$ & 3.884561 & 1.415147 & -1.0 \\
\hline $\mathrm{H}$ & 2.392863 & 2.373864 & -0.987918 \\
\hline $\mathrm{C}$ & 3.189012 & -1.233019 & 0.472604 \\
\hline $\mathrm{H}$ & 4.147732 & -1.234318 & -0.060622 \\
\hline $\mathrm{H}$ & 3.380861 & -0.983330 & 1.52068 \\
\hline
\end{tabular}




$\begin{array}{cccc}\mathrm{H} & 2.784834 & -2.250437 & 0.446309 \\ \mathrm{C} & -0.885386 & -2.583802 & 0.302333 \\ \mathrm{H} & -0.954302 & -3.377314 & -0.454259 \\ \mathrm{H} & 0.161679 & -2.390870 & 0.533790 \\ \mathrm{H} & -1.377351 & -2.953704 & 1.223612 \\ \mathrm{C} & -2.912526 & -1.605109 & -0.440432 \\ \mathrm{H} & -3.375560 & -0.693364 & -0.815698 \\ \mathrm{H} & -3.036445 & -2.409875 & -1.178608 \\ \mathrm{H} & -3.446799 & -1.910916 & 0.480507 \\ \mathrm{C} & -1.389196 & 1.341033 & -1.543486 \\ \mathrm{H} & -2.006065 & 1.985616 & -2.186953 \\ \mathrm{H} & -0.389471 & 1.802535 & -1.451387 \\ \mathrm{H} & -1.276487 & 0.364930 & -2.016895 \\ \mathrm{C} & -2.196278 & 2.462958 & 0.390706 \\ \mathrm{H} & -2.672294 & 2.329078 & 1.366403 \\ \mathrm{H} & -1.237989 & 2.990521 & 0.549116 \\ \mathrm{H} & -2.837632 & 3.105965 & -0.228615 \\ \mathrm{~F} & 1.020383 & 0.593361 & 2.154756 \\ & & & \\ \mathrm{H} \mathrm{F} & & & \\ \mathrm{C} & -0.027314 & 3.976616 & -0.798750 \\ \mathrm{C} & -0.252084 & 3.724281 & 0.560776 \\ \mathrm{C} & -0.233123 & 2.435480 & 1.072019 \\ \mathrm{C} & 0.000000 & 1.293414 & 0.243206 \\ \mathrm{C} & 0.275221 & 1.582850 & -1.130104 \\ \mathrm{C} & 0.250609 & 2.881106 & -1.623407 \\ \mathrm{H} & -0.052667 & 4.989129 & -1.196184 \\ \mathrm{H} & -0.449476 & 4.555023 & 1.240208 \\ \mathrm{H} & -0.409750 & 2.274909 & 2.131113 \\ \mathrm{H} & 0.474990 & 3.041487 & -2.679099 \\ \mathrm{C} & 0.000000 & 0.000000 & 0.800389 \\ \mathrm{C} & 0.000000 & -1.293414 & 0.243206 \\ \mathrm{C} & 0.233123 & -2.435480 & 1.072019 \\ \mathrm{C} & 0.252084 & -3.724281 & 0.560776 \\ \mathrm{C} & 0.027314 & -3.976616 & -0.798750 \\ \mathrm{C} & -0.250609 & -2.881106 & -1.623407 \\ \mathrm{C} & -0.275221 & -1.582850 & -1.130104 \\ \mathrm{H} & 0.409750 & -2.274909 & 2.131113 \\ \mathrm{H} & 0.449476 & -4.555023 & 1.240208 \\ \mathrm{H} & -0.474990 & -3.989129 & -1.196184 \\ \mathrm{H} & -0.578790 & -2.679099 \\ \mathrm{H} & 0.780104 & -1.794376 \\ \mathrm{H} & 0.000000 & 2.212162\end{array}$

$49 \mathrm{~F}$ 


$\begin{array}{lrrr}\text { C } & -0.124260 & -1.091521 & -0.419466 \\ \text { C } & 0.838547 & 1.398124 & 0.467630 \\ \text { C } & -0.503823 & 1.524345 & 0.505491 \\ \text { H } & 1.506150 & 2.134615 & 0.919329 \\ \text { H } & -0.998665 & 2.405333 & 0.921898 \\ \text { P } & 1.446339 & -0.204617 & -0.294282 \\ \text { P } & -1.360259 & 0.224171 & -0.503026 \\ \text { H } & 1.528643 & 0.283483 & -1.634240 \\ \text { H } & -2.340671 & -0.240144 & 0.418665 \\ \text { F } & -0.249938 & -1.762477 & 0.890227\end{array}$

$\begin{array}{crrr}50 \mathrm{~F} & & & \\ \mathrm{C} & -0.911440 & 0.058379 & -0.286641 \\ \mathrm{C} & 1.545503 & -0.731747 & 0.338252 \\ \mathrm{C} & 1.598800 & 0.625048 & -0.384561 \\ \mathrm{H} & 1.653007 & -0.547106 & 1.413156 \\ \mathrm{H} & 1.649464 & 0.479934 & -1.468975 \\ \mathrm{P} & -0.121464 & -1.512462 & 0.054488 \\ \mathrm{P} & 0.022539 & 1.517218 & 0.037430 \\ \mathrm{H} & 0.237808 & -2.057707 & -1.244434 \\ \mathrm{H} & 0.471724 & 1.657771 & 1.455588 \\ \mathrm{H} & 2.470609 & 1.214574 & -0.068232 \\ \mathrm{H} & 2.359505 & -1.406060 & 0.034912 \\ \mathrm{~F} & -2.306158 & 0.097462 & 0.055212 \\ & & & \\ 51 \mathrm{~F} & & & \\ \mathrm{C} & 0.246413 & 0.055191 & 0.707467 \\ \mathrm{C} & 0.246413 & 0.055191 & -0.707467 \\ \mathrm{C} & 0.024908 & 1.233451 & -1.413552 \\ \mathrm{C} & -0.218688 & 2.417984 & -0.697604 \\ \mathrm{C} & -0.218688 & 2.417984 & 0.697604 \\ \mathrm{C} & 0.024908 & 1.233451 & 1.413552 \\ \mathrm{C} & 0.347644 & -2.189379 & 0.000000 \\ \mathrm{H} & 0.012654 & 1.233457 & -2.503541 \\ \mathrm{H} & 0.012654 & 1.233457 & 2.503541 \\ \mathrm{H} & 0.219393 & -1.553958 & 1.988545 \\ \mathrm{H} & 0.219393 & -1.553958 & -1.988545 \\ \mathrm{~N} & 0.600286 & -1.231785 & 1.104695 \\ \mathrm{~N} & 0.600286 & -1.231785 & -1.104695 \\ \mathrm{H} & -0.407561 & 3.344345 & -1.239078 \\ \mathrm{H} & -0.407561 & 3.344345 & 1.239078 \\ \mathrm{~F} & -1.196714 & -2.238438 & 0.000000 \\ & & & \\ 52 \mathrm{~F} & & & \\ \mathrm{C} & -0.000517 & 1.194578 & -0.521960 \\ \mathrm{C} & -0.968674 & 0.021535 & -0.660221\end{array}$




$\begin{array}{rrrr}\mathrm{C} & 1.059678 & -0.830796 & 0.305326 \\ \mathrm{C} & 1.156513 & 0.702972 & 0.393231 \\ \mathrm{H} & 0.388673 & 1.458486 & -1.515088 \\ \mathrm{H} & -0.496027 & 2.080620 & -0.109507 \\ \mathrm{H} & -0.438809 & -1.944517 & -0.594997 \\ \mathrm{H} & 0.779680 & -1.250378 & 1.282786 \\ \mathrm{H} & 2.014318 & -1.293899 & 0.007612 \\ \mathrm{H} & 0.993557 & 1.035502 & 1.422449 \\ \mathrm{H} & 2.144130 & 1.069942 & 0.081917 \\ \mathrm{~N} & 0.019195 & -1.044169 & -0.729843 \\ \mathrm{~F} & -1.444654 & -0.041813 & 0.826164\end{array}$

$1 \mathrm{H}$

$\begin{array}{llll}\text { C } & 0.000000 & 0.684742 & -0.974396\end{array}$

$\begin{array}{llll}\text { C } & 0.000000 & -0.684742 & -0.974396\end{array}$

$\begin{array}{llll}\mathrm{H} & 0.000000 & 2.035163 & 0.676005\end{array}$

$\mathrm{H} \quad 0.000000 \quad 1.393534 \quad-1.788658$

$\mathrm{H} \quad 0.000000 \quad-1.393534 \quad-1.788658$

$\begin{array}{llll}\mathrm{H} & 0.000000 & -2.035163 & 0.676005\end{array}$

$\begin{array}{llll}\text { C } & 0.000000 & 0.000000 & 1.145213\end{array}$

$\begin{array}{llll}\mathrm{N} & 0.000000 & 1.072374 & 0.344390\end{array}$

$\mathrm{N} \quad 0.000000 \quad-1.072374 \quad 0.344390$

$\begin{array}{llll}\mathrm{H} & 0.000000 & 0.000000 & 2.225320\end{array}$

$2 \mathrm{H}$

$\begin{array}{llll}\text { C } & -0.000026 & 0.775544 & -0.980726\end{array}$

$\begin{array}{llll}\text { C } & 0.000026 & -0.775544 & -0.980726\end{array}$

$\begin{array}{llll}\text { C } & 0.000000 & 0.000000 & 1.192079\end{array}$

$\mathrm{H} \quad 0.890659 \quad 1.194480 \quad-1.451524$

$\mathrm{H} \quad-0.890769 \quad 1.194417 \quad-1.451473$

$\mathrm{H} \quad 0.890769-1.194417 \quad-1.451473$

$\mathrm{H} \quad-0.890659 \quad-1.194480 \quad-1.451524$

$\mathrm{N} \quad 0.000000 \quad-1.092030 \quad 0.461169$

$\begin{array}{llll}\mathrm{N} & 0.000000 & 1.092030 & 0.461169\end{array}$

$\mathrm{H} \quad-0.000001 \quad-2.031570 \quad 0.844685$

$\mathrm{H} \quad 0.000001 \quad 2.031570 \quad 0.844685$

$\begin{array}{llll}\mathrm{H} & 0.000000 & 0.000000 & 2.276499\end{array}$

$3 \mathrm{H}$

$\begin{array}{llll}\text { C } & 0.000000 & 0.685079 & -1.232162\end{array}$

C $\quad 0.000000 \quad-0.685079 \quad-1.232162$

$\mathrm{H} \quad 0.000000 \quad 1.391362 \quad-2.049760$

H $\quad 0.000000 \quad-1.391362 \quad-2.049760$

$\begin{array}{llll}\text { C } & 0.000000 & 0.000000 & 0.867466\end{array}$

$\begin{array}{llll}\mathrm{N} & 0.000000 & 1.086971 & 0.081629\end{array}$

$\mathrm{N} \quad 0.000000 \quad-1.086971 \quad 0.081629$ 


\begin{tabular}{|c|c|c|c|}
\hline $\mathrm{C}$ & 0.000000 & 2.478923 & \\
\hline $\mathrm{H}$ & 0.895394 & 2.978734 & 0.180612 \\
\hline $\mathrm{H}$ & 0.895394 & 2.978734 & 0.180612 \\
\hline $\mathrm{H}$ & 0.000000 & 2.479759 & 195 \\
\hline $\mathrm{C}$ & 0.000000 & -2.478923 & 240 \\
\hline $\mathrm{H}$ & -0.895394 & -2.978734 & 612 \\
\hline $\mathrm{H}$ & 0.895394 & -2.978734 & 0612 \\
\hline $\mathrm{H}$ & 0.000000 & -2.479759 & 195 \\
\hline $\mathrm{H}$ & 0.000000 & 0.000000 & 1.9 \\
\hline \multicolumn{4}{|l|}{18} \\
\hline $\mathrm{C}$ & -0.000482 & -0.7 & -1.2 \\
\hline $\mathrm{C}$ & 0.000482 & $0.7^{\prime}$ & \\
\hline $\mathrm{C}$ & 0.000000 & 0.000000 & \\
\hline $\mathrm{H}$ & -0.8 & -1.1 & 668 \\
\hline $\mathrm{H}$ & 0.888436 & -1.1 & -1.7 \\
\hline $\mathrm{H}$ & -0.8 & & \\
\hline $\mathrm{H}$ & 0.8 & 1.1 & -1. \\
\hline $\mathrm{N}$ & 000 & 1.1 & 0.1 \\
\hline $\mathrm{N}$ & 0.0 & -1.1 & \\
\hline $\mathrm{C}$ & 0.000469 & $2.4^{\prime}$ & 0.6 \\
\hline $\mathrm{H}$ & 0.0 & 2.4 & \\
\hline $\mathrm{H}$ & 0.89 & 2.99 & 172 \\
\hline $\mathrm{H}$ & -0.89 & 2.992 & \\
\hline $\mathrm{C}$ & -0.0 & -2.4 & \\
\hline $\mathrm{H}$ & -0.8 & & \\
\hline $\mathrm{H}$ & 0.8 & -2.9 & \\
\hline $\mathrm{H}$ & -0.00 & -2.4 & 009 \\
\hline $\mathrm{H}$ & $0 .($ & 0.0 & 178 \\
\hline \multicolumn{4}{|l|}{$5 \mathrm{H}$} \\
\hline $\mathrm{C}$ & 0.0 & 0.6 & -0 . \\
\hline $\mathrm{C}$ & 0. & -0.6 & -0 . \\
\hline $\mathrm{C}$ & 0.0 & 0.0 & \\
\hline $\mathrm{N}$ & 0.0 & 1.0 & \\
\hline $\mathrm{N}$ & 0.0 & -1.06 & 086 \\
\hline $\mathrm{C}$ & 0.000000 & -1.684 & -1. \\
\hline $\mathrm{H}$ & 0.8 & -2.320 & -1.3 \\
\hline $\mathrm{H}$ & -0.888523 & -2.320719 & -1.3 \\
\hline $\mathrm{H}$ & 0.000000 & -1.170439 & -2.3 \\
\hline $\mathrm{C}$ & 0.000000 & 1.684834 & 3660 \\
\hline $\mathrm{H}$ & -0.88 & 2.320719 & 2398 \\
\hline $\mathrm{H}$ & 0.888523 & 2.320719 & -1.372398 \\
\hline $\mathrm{H}$ & 0.000000 & & -2.3 \\
\hline $\mathrm{H}$ & 0.000000 & -2.031533 & 1.353104 \\
\hline $\mathrm{H}$ & 0.000000 & 2.031533 & 1.35310 \\
\hline
\end{tabular}




\begin{tabular}{|c|c|c|c|}
\hline $\mathrm{H}$ & 0.000000 & 0.000000 & 29 \\
\hline \multicolumn{4}{|l|}{$6 \mathrm{H}$} \\
\hline $\mathrm{C}$ & 0.000000 & 0.690756 & -0.661679 \\
\hline $\mathrm{C}$ & 0.000000 & -0.690756 & -0.661679 \\
\hline C & 0.000000 & 0.000000 & 1.451499 \\
\hline $\mathrm{N}$ & 0.000000 & 1.083671 & 0.663030 \\
\hline $\mathrm{N}$ & 0.000000 & -1.083671 & 0.663030 \\
\hline C & 0.000000 & 2.468519 & 1.139156 \\
\hline $\mathrm{H}$ & 0.893849 & & 417 \\
\hline $\mathrm{H}$ & -0.893849 & 2.977068 & 0.777417 \\
\hline $\mathrm{H}$ & 0.000000 & 2.460249 & 8340 \\
\hline C & 0.000000 & -2.468519 & 9156 \\
\hline $\mathrm{H}$ & -0.893849 & -2.977068 & 7417 \\
\hline $\mathrm{H}$ & 0.893849 & 7068 & 417 \\
\hline $\mathrm{H}$ & 0.000000 & -2.460249 & 2.228340 \\
\hline $\mathrm{C}$ & 0.000000 & -1.673157 & 9255 \\
\hline $\mathrm{H}$ & 0.887142 & -2.3 & -1. \\
\hline $\mathrm{H}$ & -0.8 & & \\
\hline $\mathrm{H}$ & 000 & -1.1 & -2. \\
\hline $\mathrm{C}$ & 0.000000 & 1.67 & -1.7 \\
\hline $\mathrm{H}$ & -0.8 & 84 & -1. \\
\hline $\mathrm{H}$ & 0.8 & 84 & 858 \\
\hline $\mathrm{H}$ & 000 & 72 & 4537 \\
\hline$H$ & 0.000000 & 0.000000 & 2.532081 \\
\hline \multicolumn{4}{|l|}{$7 \mathrm{H}$} \\
\hline $\mathrm{C}$ & & & \\
\hline $\mathrm{C}$ & 0.0 & 0.6 & -0 \\
\hline $\mathrm{C}$ & 0.000000 & -0.6 & -0. \\
\hline $\mathrm{H}$ & 00 & 1.4 & 1305 \\
\hline $\mathrm{H}$ & 0.000000 & -1.4 & -1.7 \\
\hline $\mathrm{O}$ & 0000 & 1.0 & 3421 \\
\hline $\mathrm{O}$ & 0.000000 & -1.0 & 8421 \\
\hline $\mathrm{H}$ & 0.000000 & 0.000000 & 2.179742 \\
\hline \multicolumn{4}{|l|}{$8 \mathrm{H}$} \\
\hline $\mathrm{C}$ & 0.000000 & 0.000000 & 575 \\
\hline S & 0.000000 & 0.685036 & -1.2 \\
\hline $\mathrm{C}$ & 0.000000 & -0.685036 & -1.230244 \\
\hline 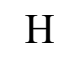 & 0.000000 & 1.307977 & -2.119162 \\
\hline $\mathrm{H}$ & 0.000000 & -1.307977 & -2.119162 \\
\hline $\mathrm{S}$ & 0.000000 & 1.427757 & 0.302022 \\
\hline S & 0.000000 & -1.427757 & 0.302022 \\
\hline $\mathrm{H}$ & 0.000000 & 0.000000 & 2.26507 \\
\hline
\end{tabular}




\begin{tabular}{|c|c|c|c|}
\hline \multicolumn{4}{|l|}{$9 \mathrm{H}$} \\
\hline $\mathrm{C}$ & 0.945289 & -0.707304 & 0.145676 \\
\hline $\mathrm{C}$ & -0.375332 & -0.000410 & -0.535985 \\
\hline $\mathrm{C}$ & -1.509787 & 0.000084 & 0.206531 \\
\hline $\mathrm{C}$ & 0.945184 & 0.707552 & 0.145154 \\
\hline $\mathrm{H}$ & 0.714843 & -1.257698 & 1.051082 \\
\hline $\mathrm{H}$ & 1.452116 & -1.259344 & -0.640532 \\
\hline $\mathrm{H}$ & -1.481221 & 0.000514 & 1.294113 \\
\hline $\mathrm{H}$ & -2.490835 & 0.000099 & -0.262642 \\
\hline $\mathrm{H}$ & 0.714590 & 1.258602 & 1.050120 \\
\hline $\mathrm{H}$ & 1.451787 & 1.259102 & -0.641539 \\
\hline $\mathrm{H}$ & -0.393405 & -0.000810 & -1.618856 \\
\hline \multicolumn{4}{|c|}{$10 \mathrm{H}$} \\
\hline $\mathrm{C}$ & -1.338103 & -1.397608 & 0.000000 \\
\hline $\mathrm{C}$ & 1.1 & 1.4 & \\
\hline $\mathrm{C}$ & 0.000000 & 187 & 0.000000 \\
\hline $\mathrm{C}$ & -0.106715 & -0.7 & 0.000000 \\
\hline $\mathrm{H}$ & 1.088152 & 2.496668 & 0.000000 \\
\hline $\mathrm{H}$ & 2.109312 & 0.956005 & 0.000000 \\
\hline $\mathrm{H}$ & -1.156288 & -2.507514 & 0.000000 \\
\hline $\mathrm{H}$ & 0.884380 & -1.2 & 00 \\
\hline $\mathrm{H}$ & -0.9 & 740 & 000 \\
\hline \multicolumn{4}{|c|}{$11 \mathrm{H}$} \\
\hline $\mathrm{C}$ & 0.00 & 10 & $-0 .{ }^{2}$ \\
\hline $\mathrm{C}$ & 0.000000 & 000 & 006 \\
\hline $\mathrm{C}$ & 0.000000 & -0.76 & 257 \\
\hline $\mathrm{H}$ & -0.913250 & 1.319917 & 9293 \\
\hline $\mathrm{H}$ & 0.9 & 1.319917 & 293 \\
\hline $\mathrm{H}$ & 0.91 & -1.319917 & -0.609293 \\
\hline $\mathrm{H}$ & -0.91 & -1.3 & -0.6 \\
\hline $\mathrm{H}$ & 0.000000 & 0.000000 & 1.918215 \\
\hline \multicolumn{4}{|l|}{$12 \mathrm{H}$} \\
\hline $\mathrm{C}$ & 0.000 & 00 & 14 \\
\hline $\mathrm{C}$ & 0.000000 & 740 & 757 \\
\hline $\mathrm{C}$ & 0.000 & -0.683740 & -0.394757 \\
\hline $\mathrm{H}$ & 0.000000 & -1.621847 & -0.936372 \\
\hline $\mathrm{H}$ & & & -0.936372 \\
\hline $\mathrm{H}$ & 0.000000 & 0.000000 & 1.872746 \\
\hline \multicolumn{4}{|c|}{$13 \mathrm{H}$} \\
\hline $\mathrm{C}$ & 0.00 & 0.0 & \\
\hline $\mathrm{H}$ & 0.000000 & 1.088272 & 0.000000 \\
\hline $\mathrm{H}$ & 0.942471 & -0.544136 & 0.000000 \\
\hline
\end{tabular}




\begin{tabular}{|c|c|c|c|}
\hline $\mathrm{H}$ & -0.942471 & -0.544136 & 0.000000 \\
\hline \multicolumn{4}{|c|}{$14 \mathrm{H}$} \\
\hline $\mathrm{C}$ & 0.000000 & 0.000000 & -0.599629 \\
\hline $\mathrm{H}$ & 0.000000 & 0.972255 & -1.093723 \\
\hline $\mathrm{F}$ & 0.000000 & 0.000000 & 0.642802 \\
\hline $\mathrm{H}$ & 0.000000 & -0.972255 & -1.093723 \\
\hline \multicolumn{4}{|c|}{$15 \mathrm{H}$} \\
\hline $\mathrm{C}$ & 0.000000 & 0.000000 & 0.415503 \\
\hline $\mathrm{F}$ & 0.000000 & 1.066609 & -0.222283 \\
\hline $\mathrm{F}$ & 0.000000 & -1.066609 & -0.222283 \\
\hline$U$ & 0.000000 & 0.000000 & 1.508066 \\
\hline \multicolumn{4}{|c|}{$16 \mathrm{H}$} \\
\hline $\mathrm{C}$ & -0.690201 & 0.000004 & -0.062474 \\
\hline 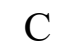 & & 03 & 2484 \\
\hline $\mathrm{H}$ & -1.244812 & 0.935744 & -0.073666 \\
\hline $\mathrm{H}$ & -1.244826 & -0.935728 & -0.073725 \\
\hline $\mathrm{H}$ & 0.000043 & 0056 & 1534 \\
\hline $\mathrm{H}$ & 1.244812 & -0.93 & -0.073702 \\
\hline $\mathrm{H}$ & 1.244821 & 746 & -0.073694 \\
\hline \multicolumn{4}{|c|}{$17 \mathrm{H}$} \\
\hline $\mathrm{C}$ & 0.00 & 1.2 & 6300 \\
\hline C & & & \\
\hline $\mathrm{H}$ & 0.557298 & 2.045098 & 0.348696 \\
\hline $\mathrm{H}$ & 0.218027 & 1.244833 & -1.263122 \\
\hline $\mathrm{H}$ & -1.068559 & 1.579812 & -0.072482 \\
\hline C & 0.000000 & -1.277375 & -0.196300 \\
\hline $\mathrm{H}$ & -0.557298 & -2.045098 & 0.348696 \\
\hline $\mathrm{H}$ & -0.218027 & -1.24 & -1.263122 \\
\hline $\mathrm{H}$ & 1.068559 & -1.579812 & -0.072482 \\
\hline $\mathrm{H}$ & 0.000000 & 0.000000 & 1.555089 \\
\hline \multicolumn{4}{|l|}{$18 \mathrm{H}$} \\
\hline $\mathrm{C}$ & 0.00 & 736 & 359 \\
\hline 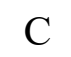 & 0.34 & 1.231168 & -0.425494 \\
\hline $\mathrm{H}$ & 0.517212 & 3.367055 & -0.201506 \\
\hline $\mathrm{H}$ & -1.073341 & 2.724936 & 0.221741 \\
\hline $\mathrm{H}$ & 0.296615 & 2.517487 & 1.334892 \\
\hline $\mathrm{C}$ & 0.000000 & 0.000000 & 0.227029 \\
\hline $\mathrm{H}$ & 0.133951 & 1.216782 & -1.497893 \\
\hline $\mathrm{H}$ & 1.462569 & 1.068859 & -0.352735 \\
\hline $\mathrm{C}$ & -0.349962 & -1.231168 & -0.425494 \\
\hline $\mathrm{H}$ & -1.462569 & -1.068859 & -0.352735 \\
\hline
\end{tabular}




$\begin{array}{cccc}\mathrm{H} & -0.133951 & -1.216782 & -1.497893 \\ \mathrm{C} & 0.000000 & -2.539736 & 0.284359 \\ \mathrm{H} & -0.296615 & -2.517487 & 1.334892 \\ \mathrm{H} & -0.517212 & -3.367055 & -0.201506 \\ \mathrm{H} & 1.073341 & -2.724936 & 0.221741 \\ \mathrm{H} & 0.000000 & 0.000000 & 1.322449\end{array}$

$19 \mathrm{H}$

$\begin{array}{lrcc}\mathrm{C} & -0.502710 & -0.008514 & -0.000014 \\ \mathrm{C} & 0.795908 & -0.730834 & 0.000068 \\ \mathrm{~F} & 1.789550 & 0.189611 & -0.000066 \\ \mathrm{~F} & -0.571988 & 1.238981 & 0.000035 \\ \mathrm{~F} & -1.595484 & -0.630054 & -0.000042 \\ \mathrm{H} & 0.821060 & -1.375513 & -0.892632 \\ \mathrm{H} & 0.821049 & -1.375235 & 0.892971\end{array}$

$20 \mathrm{H}$

$\begin{array}{lrrr}\mathrm{C} & -1.349776 & -0.142278 & 0.143765 \\ \mathrm{C} & -0.101623 & 0.496740 & 0.664871 \\ \mathrm{~F} & 0.837213 & -0.126358 & -1.388727 \\ \mathrm{~F} & 2.201235 & 0.464532 & 0.218046 \\ \mathrm{~F} & 1.146522 & -1.454388 & 0.317637 \\ \mathrm{~F} & -0.160255 & 1.812928 & 0.354647 \\ \mathrm{~F} & -1.604212 & -1.353161 & 0.345665 \\ \mathrm{~F} & -2.177657 & 0.502420 & -0.532974 \\ \mathrm{C} & 1.090934 & -0.177303 & -0.071396 \\ \mathrm{H} & -0.022819 & 0.323292 & 1.747912\end{array}$

$21 \mathrm{H}$

$\begin{array}{llll}\text { C } & -1.180771 & -0.000050 & -0.007969\end{array}$

SI $\quad 0.640554 \quad-0.000003 \quad-0.004690$

$\begin{array}{llll}\mathrm{H} & 1.407970 & 1.251774 & 0.016501\end{array}$

$\mathrm{H} \quad-1.523740 \quad 0.001119 \quad 1.039330$

$\begin{array}{llll}\mathrm{H} & 1.408328 & -1.251558 & 0.016599\end{array}$

$\mathrm{H} \quad-1.587816 \quad-0.898919 \quad-0.478628$

$\mathrm{H} \quad-1.587871 \quad 0.897927 \quad-0.480322$

$\begin{array}{cccc}{ }^{3} 21 \mathrm{H} & & & \\ \mathrm{C} & -1.511228 & 0.000000 & -0.071757 \\ \mathrm{SI} & 0.712687 & 0.000000 & 0.001597 \\ \mathrm{H} & 0.880170 & 1.263913 & -0.725169 \\ \mathrm{H} & 0.915183 & 0.000066 & 1.455561 \\ \mathrm{H} & 0.880171 & -1.263978 & -0.725055 \\ \mathrm{H} & -1.792884 & -1.011509 & 0.201419 \\ \mathrm{H} & -1.792886 & 1.011508 & 0.201420\end{array}$




\begin{tabular}{|c|c|c|c|}
\hline \multicolumn{4}{|l|}{$22 \mathrm{H}$} \\
\hline $\mathrm{C}$ & -0.114586 & -0.859871 & 0.000245 \\
\hline $\mathrm{H}$ & 611 & -0.781100 & \\
\hline $\mathrm{H}$ & 1.505737 & 1.051363 & -1.220422 \\
\hline $\mathrm{H}$ & 1.505529 & 1.052478 & \\
\hline S & 1.534443 & 0.220718 & -0.000088 \\
\hline SI & -1.571688 & & -0.0 \\
\hline $\mathrm{H}$ & -2.158850 & 036 & 738 \\
\hline $\mathrm{H}$ & -2.159822 & & -1.2 \\
\hline $\mathrm{H}$ & -0.0 & -1.4 & 0719 \\
\hline L & -0.0 & -1 & 0.90 \\
\hline \multicolumn{4}{|l|}{$23 \mathrm{H}$} \\
\hline $\mathrm{C}$ & -1.1 & -0. & -0.0 \\
\hline S & 0.4 & $-0 .($ & \\
\hline 4 & 0.4 & & -0. \\
\hline $\mathrm{H}$ & 1.5 & 2.2 & \\
\hline $\mathrm{H}$ & -0.0 & & -0 . \\
\hline $\mathrm{H}$ & -0.0 & 2.2 & 88 \\
\hline $\mathrm{C}$ & 2.0 & -0.9 & \\
\hline $\mathrm{H}$ & 978 & -0.6 & -0 . \\
\hline $\mathrm{H}$ & 2.6 & -0.6 & \\
\hline $\mathrm{H}$ & & -1. & 07 \\
\hline $\mathrm{C}$ & -2.3 & -0.0 & \\
\hline $\mathrm{H}$ & -3.2 & -0 & 170 \\
\hline $\mathrm{H}$ & -2.4 & & \\
\hline $\mathrm{H}$ & -2.4 & 0.5 & -0.8 \\
\hline $\mathrm{H}$ & -1.0 & -1.6 & 74 \\
\hline $\mathrm{H}$ & -1.0 & -1 & 736 \\
\hline \multicolumn{4}{|l|}{$24 \mathrm{H}$} \\
\hline $\mathrm{C}$ & -0.0 & -0 & \\
\hline S & -1.5 & $-0 .($ & \\
\hline $\mathrm{C}$ & 0.2 & -2.1 & \\
\hline $\mathrm{H}$ & 903 & -2.2 & \\
\hline $\mathrm{H}$ & -0.3 & -2.5 & 520 \\
\hline $\mathrm{H}$ & 0.08 & -2.6 & 027 \\
\hline $\mathrm{C}$ & -2.5 & 1.4 & 43 \\
\hline $\mathrm{H}$ & -2.7 & 003 & 441 \\
\hline $\mathrm{H}$ & & & 71 \\
\hline $\mathrm{H}$ & -1.9 & 1.9 & 033 \\
\hline $\mathrm{C}$ & -2.26 & -0.9 & 6210 \\
\hline $\mathrm{H}$ & -3.3 & -1.1 & 4892 \\
\hline $\mathrm{H}$ & -2.2 & -0.2 & 567 \\
\hline $\mathrm{H}$ & -1.745615 & -1.870344 & -1.488162 \\
\hline SI & 1.289043 & 0.298647 & -0.156415 \\
\hline
\end{tabular}




$\begin{array}{crrr}\mathrm{C} & 1.716231 & -0.854624 & -1.565386 \\ \mathrm{H} & 2.441865 & -0.365156 & -2.223065 \\ \mathrm{H} & 2.171397 & -1.784518 & -1.215769 \\ \mathrm{H} & 0.849221 & -1.112919 & -2.181225 \\ \mathrm{C} & 2.732319 & 0.669483 & 0.967414 \\ \mathrm{H} & 3.547621 & 1.116843 & 0.389930 \\ \mathrm{H} & 2.459196 & 1.374414 & 1.757569 \\ \mathrm{H} & 3.122513 & -0.236438 & 1.439365 \\ \mathrm{C} & 0.551283 & 1.892773 & -0.851119 \\ \mathrm{H} & 0.223037 & 2.594461 & -0.078483 \\ \mathrm{H} & 1.336267 & 2.403189 & -1.420903 \\ \mathrm{H} & -0.270917 & 1.749340 & -1.564432 \\ \mathrm{H} & 0.050866 & -0.094206 & 1.950134 \\ & & & \\ { }^{3} 24 \mathrm{H} & & & \\ \mathrm{C} & 0.000000 & 0.012021 & 0.000003 \\ \mathrm{SI} & -1.842862 & 0.012023 & 0.000000 \\ \mathrm{C} & -2.471288 & -0.510115 & -1.698196 \\ \mathrm{H} & -2.124971 & -1.514522 & -1.957784 \\ \mathrm{H} & -3.565793 & -0.517281 & -1.721847 \\ \mathrm{H} & -2.124811 & 0.174847 & -2.477262 \\ \mathrm{C} & -2.471292 & -1.197589 & 1.301283 \\ \mathrm{H} & -2.124977 & -0.920194 & 2.300920 \\ \mathrm{H} & -3.565797 & -1.214488 & 1.319312 \\ \mathrm{H} & -2.124814 & -2.214761 & 1.097623 \\ \mathrm{C} & -2.471289 & 1.743773 & 0.396912 \\ \mathrm{H} & -3.565794 & 1.767845 & 0.402501 \\ \mathrm{H} & -2.124838 & 2.075972 & 1.379654 \\ \mathrm{H} & -2.124945 & 2.470792 & -0.343118 \\ \mathrm{SI} & 1.842862 & 0.012023 & 0.000000 \\ \mathrm{C} & 2.471287 & 1.227129 & -1.296154 \\ \mathrm{H} & 3.565792 & 1.243931 & -1.314277 \\ \mathrm{H} & 2.124780 & 0.954087 & -2.296926 \\ \mathrm{H} & 2.124999 & 2.243467 & -1.088074 \\ \mathrm{C} & 2.471290 & -1.718031 & -0.404236 \\ \mathrm{H} & 3.565795 & -1.742120 & -0.409747 \\ \mathrm{H} & 2.124803 & -2.448202 & 0.332624 \\ \mathrm{H} & 2.124984 & -2.046007 & -1.388441 \\ \mathrm{C} & 2.471291 & 0.526972 & 1.700390 \\ \mathrm{H} & 2.124996 & -0.161393 & 2.476525 \\ \mathrm{H} & 3.565796 & 0.534257 & 1.724002 \\ \mathrm{H} & 2.124793 & 1.530192 & 1.964311 \\ \mathrm{H} & 0.000000 & -1.058030 & -0.000005 \\ & & & \\ 25 \mathrm{H} & & & \\ \mathrm{C} & -0.439034 & 0.586237 & -0.000086 \\ & & & \end{array}$




\begin{tabular}{|c|c|c|c|}
\hline $\mathrm{O}$ & -0.551581 & -0.659759 & 0.000030 \\
\hline $\mathrm{C}$ & 0.900214 & 0.086447 & 0.000005 \\
\hline $\mathrm{H}$ & -1.183483 & 1.382113 & 0.000159 \\
\hline $\mathrm{H}$ & 1.414554 & -0.070200 & -0.943704 \\
\hline $\mathrm{H}$ & 1.414491 & -0.069943 & 0.943791 \\
\hline \multicolumn{4}{|c|}{${ }^{3} 25 \mathrm{H}$} \\
\hline $\mathrm{C}$ & 0.000000 & 0.436334 & 0.000000 \\
\hline $\mathrm{O}$ & -1.194251 & 0.046719 & 0.000000 \\
\hline $\mathrm{C}$ & 1.076821 & -0.480606 & 0.000000 \\
\hline $\mathrm{H}$ & 0.112386 & 1.531638 & 0.000000 \\
\hline $\mathrm{H}$ & 0.893340 & -1.549366 & 0.000000 \\
\hline $\mathrm{H}$ & 2.087355 & -0.090390 & 0.000000 \\
\hline \multicolumn{4}{|l|}{$26 \mathrm{H}$} \\
\hline $\mathrm{C}$ & 0.898187 & 0.474197 & -0.099193 \\
\hline $\mathrm{O}$ & 1.384296 & -0.624566 & 0.080911 \\
\hline $\mathrm{H}$ & 1.463488 & 1.375987 & -0.369815 \\
\hline $\mathrm{C}$ & -0.622300 & 0.643629 & 0.033907 \\
\hline $\mathrm{C}$ & -1.437602 & -0.464076 & -0.122640 \\
\hline $\mathrm{H}$ & -0.997251 & -0.116018 & 1.042523 \\
\hline $\mathrm{H}$ & -2.520871 & -0.377935 & -0.075440 \\
\hline $\mathrm{H}$ & -0.984177 & -1.433330 & -0.328751 \\
\hline $\mathrm{H}$ & -1.065268 & 1.625327 & 0.211751 \\
\hline \multicolumn{4}{|l|}{$27 \mathrm{H}$} \\
\hline $\mathrm{C}$ & 0.000000 & 0.000000 & 0.601951 \\
\hline CL & 0.000000 & 1.432493 & -0.156032 \\
\hline CL & 0.000000 & -1.432493 & -0.156032 \\
\hline $\mathrm{H}$ & 0.000000 & 0.000000 & 1.693371 \\
\hline \multicolumn{4}{|l|}{$28 \mathrm{H}$} \\
\hline$C$ & 0.000000 & 0.665751 & 0.000000 \\
\hline CL & -1.391682 & -0.133468 & 0.000000 \\
\hline $\mathrm{C}$ & 1.248781 & 0.009460 & 0.000000 \\
\hline $\mathrm{N}$ & 2.315188 & -0.505853 & 0.000000 \\
\hline $\mathrm{H}$ & -0.040409 & 1.758654 & 0.000000 \\
\hline \multicolumn{4}{|l|}{$29 \mathrm{H}$} \\
\hline $\mathrm{C}$ & 0.000000 & 0.637239 & 0.000000 \\
\hline CL & -1.327185 & -0.288844 & 0.000000 \\
\hline $\mathrm{N}$ & 1.214421 & 0.136822 & 0.000000 \\
\hline $\mathrm{C}$ & 2.363636 & -0.265724 & 0.000000 \\
\hline $\mathrm{H}$ & -0.120609 & 1.723499 & 0.000000 \\
\hline
\end{tabular}




$$
\begin{array}{cccc}
\mathrm{C} & -0.546440 & 0.535491 & 0.000000 \\
\mathrm{C} & 0.000000 & -0.762909 & 0.000000 \\
\mathrm{~N} & 0.410715 & -1.873949 & 0.000000 \\
\mathrm{~F} & 0.224741 & 1.525757 & 0.000000 \\
\mathrm{H} & -1.619038 & 0.750345 & 0.000000
\end{array}
$$

$$
\begin{array}{cccc}
31 \mathrm{H} & & & \\
\mathrm{C} & 0.000000 & 0.686991 & 0.000000 \\
\mathrm{~N} & -0.556864 & -0.488931 & 0.000000 \\
\mathrm{C} & -1.143111 & -1.560619 & 0.000000 \\
\mathrm{~F} & 1.259033 & 0.783179 & 0.000000 \\
\mathrm{H} & -0.574578 & 1.615675 & 0.000000
\end{array}
$$




\begin{tabular}{|c|c|c|c|}
\hline $\mathrm{C}$ & 0.000000 & 2.382222 & 0.2 \\
\hline $\mathrm{H}$ & -0.898696 & 2.893822 & -0.094000 \\
\hline $\mathrm{H}$ & 0.000000 & 2.218542 & 1.316294 \\
\hline $\mathrm{H}$ & 0.898696 & 2.893822 & -0.094000 \\
\hline $\mathrm{C}$ & 0.000000 & 0.000000 & 0.188566 \\
\hline $\mathrm{C}$ & 0.000000 & -2.382222 & 0.238983 \\
\hline $\mathrm{H}$ & -0.898696 & -2.893822 & -0.094000 \\
\hline $\mathrm{H}$ & 0.898696 & -2.893822 & -0.094000 \\
\hline $\mathrm{H}$ & 0.000000 & -2.218542 & 1.316294 \\
\hline $\mathrm{O}$ & 0.000000 & 1.085487 & -0.47 \\
\hline $\mathrm{O}$ & 0.000000 & -1.085487 & \\
\hline $\mathrm{H}$ & 0000 & 0.000000 & 66 \\
\hline \multicolumn{4}{|l|}{$37 \mathrm{H}$} \\
\hline $\mathrm{C}$ & 0.62 & 0.51 & 0.00 \\
\hline $\mathrm{N}$ & & 1.5 & \\
\hline $\mathrm{C}$ & 0.000000 & -0.756864 & 0.000000 \\
\hline $\mathrm{N}$ & -1.2 & -0.864060 & 0.000000 \\
\hline $\mathrm{H}$ & -1.898360 & -0.041016 & 0.000000 \\
\hline $\mathrm{H}$ & -1.737974 & -1.78 & 0.000000 \\
\hline $\mathrm{H}$ & 0.602281 & -1.664548 & 0.000000 \\
\hline \multicolumn{4}{|l|}{$38 \mathrm{H}$} \\
\hline $\mathrm{N}$ & 0.0 & 1.164447 & -0.17 \\
\hline $\mathrm{H}$ & 0.0 & 2.02 & \\
\hline $\mathrm{H}$ & 000 & 1.26 & -1.1 \\
\hline $\mathrm{C}$ & 0.0 & 0.00 & 0.4 \\
\hline $\mathrm{N}$ & 0.000000 & -1.16 & -0.1 \\
\hline $\mathrm{H}$ & 0.000000 & -2.021038 & 0.367952 \\
\hline $\mathrm{H}$ & 0.000000 & -1.26 & -1.18 \\
\hline $\mathrm{H}$ & 000 & 0.00 & 1.516240 \\
\hline \multicolumn{4}{|c|}{$39 \mathrm{H}$} \\
\hline $\mathrm{C}$ & -0.8 & -0.027184 & 0.017453 \\
\hline $\mathrm{C}$ & 0.3 & -0.510044 & -0.6 \\
\hline C & 1.5 & 0.332637 & -0.265178 \\
\hline $\mathrm{H}$ & 0.546425 & -1.570940 & -0.475265 \\
\hline $\mathrm{H}$ & 0.241981 & -0.378374 & -1.767920 \\
\hline $\mathrm{N}$ & 2.650080 & -0.150256 & 0.152241 \\
\hline $\mathrm{H}$ & 3.423408 & 0.456393 & 0.433702 \\
\hline $\mathrm{H}$ & 2.810545 & -1.157705 & 0.225222 \\
\hline $\mathrm{F}$ & & 1.267061 & -0.283170 \\
\hline $\mathrm{F}$ & -0.700230 & -0.123052 & 1.350005 \\
\hline $\mathrm{F}$ & -1.9 & -0.75 & -0.35 \\
\hline - & 1.444210 & 1.417202 & -0.30186 \\
\hline
\end{tabular}




$\begin{array}{cccc}40 \mathrm{H} & & & \\ \mathrm{C} & -0.643691 & -0.654709 & 0.001531 \\ \mathrm{C} & 0.599070 & 0.019124 & 0.478150 \\ \mathrm{H} & -0.988568 & -1.356957 & 0.765488 \\ \mathrm{H} & -0.478031 & -1.215208 & -0.925679 \\ \mathrm{~N} & 1.674302 & 0.129098 & -0.224944 \\ \mathrm{H} & 2.471698 & 0.681324 & 0.092638 \\ \mathrm{H} & 1.767865 & -0.324703 & -1.134619 \\ \mathrm{~N} & -1.528721 & 0.492520 & -0.113248 \\ \mathrm{H} & -1.662683 & 0.794185 & -1.072850 \\ \mathrm{H} & -2.431552 & 0.351597 & 0.328658 \\ \mathrm{H} & 0.569928 & 0.531942 & 1.435619 \\ & & & \\ 41 \mathrm{H} & & & \\ \mathrm{P} & 0.000000 & -0.510119 & 0.000000 \\ \mathrm{H} & 1.188571 & -1.233884 & 0.000000 \\ \mathrm{H} & -1.188572 & -1.233881 & 0.000000 \\ \mathrm{C} & 0.000000 & 1.127875 & 0.000000 \\ \mathrm{H} & 0.938527 & 1.676148 & 0.000000 \\ \mathrm{H} & -0.938528 & 1.676146 & 0.000000 \\ & & & \\ 42 \mathrm{H} & & & \\ \mathrm{P} & -1.562636 & 0.131496 & -0.100211 \\ \mathrm{C} & 0.000843 & -0.577252 & 0.107493 \\ \mathrm{P} & 1.561320 & 0.131392 & -0.099076 \\ \mathrm{H} & 1.568116 & 1.344371 & 0.601926 \\ \mathrm{H} & 2.432372 & -0.747590 & 0.554439 \\ \mathrm{H} & -2.423926 & -0.749149 & 0.564657 \\ \mathrm{H} & -1.563541 & 1.339842 & 0.609480 \\ \mathrm{H} & 0.001652 & -1.667286 & 0.013845 \\ & & & \\ 43 \mathrm{H} & & & \\ \mathrm{C} & -1.598668 & -0.068125 & 0.028672 \\ \mathrm{C} & -0.294432 & -0.860743 & 0.016599 \\ \mathrm{~S} & 1.084980 & -0.135428 & -0.002321 \\ \mathrm{~F} & 2.225515 & -1.109725 & -0.325433 \\ \mathrm{~F} & 1.831783 & 0.619310 & 1.154080 \\ \mathrm{~F} & 1.191126 & 1.046348 & -0.979242 \\ \mathrm{~F} & -2.276271 & -0.349049 & 1.131824 \\ \mathrm{~F} & -1.298283 & 1.248247 & 0.006980 \\ \mathrm{~F} & -2.312781 & -0.380880 & -1.040722 \\ \mathrm{H} & -0.250886 & -1.928201 & 0.238130 \\ & & & \\ 44 \mathrm{H} & & & \\ \mathrm{P} & -1.542365 & 0.145447 & 0.000000 \\ \mathrm{H} & -1.814001 & -1.219779 & 0.000000 \\ & & & \end{array}$




$\begin{array}{cccc}\mathrm{H} & -2.664336 & 0.973686 & 0.000000 \\ \mathrm{C} & 0.000000 & 0.696807 & 0.000000 \\ \mathrm{H} & 2.288785 & -0.074157 & 1.234041 \\ \mathrm{H} & 1.044539 & -1.807569 & 0.000000 \\ \mathrm{H} & 2.288785 & -0.074157 & -1.234041 \\ \mathrm{SI} & 1.563281 & -0.424601 & 0.000000 \\ \mathrm{H} & 0.105768 & 1.783846 & 0.000000\end{array}$

$\begin{array}{cccc}45 \mathrm{H} & & & \\ \mathrm{P} & -0.845976 & -0.073753 & 0.045493 \\ \mathrm{C} & 0.591019 & -0.810567 & 0.186142 \\ \mathrm{H} & 2.947514 & -0.683206 & -1.191948 \\ \mathrm{H} & 2.007205 & 1.427275 & -0.389037 \\ \mathrm{H} & 3.032972 & -0.122577 & 1.192066 \\ \mathrm{SI} & 2.280960 & 0.006978 & -0.070985 \\ \mathrm{~N} & -1.089797 & 1.530052 & 0.043809 \\ \mathrm{H} & -0.414049 & 2.158670 & 0.469205 \\ \mathrm{H} & -1.734449 & 1.956312 & -0.617274 \\ \mathrm{~N} & -2.293850 & -0.789211 & -0.128874 \\ \mathrm{H} & -2.364730 & -1.727732 & -0.512247 \\ \mathrm{H} & -3.105960 & -0.457379 & 0.386040 \\ \mathrm{H} & 0.527108 & -1.865249 & 0.453195 \\ & & & \\ 46 \mathrm{H} & & & \\ \mathrm{P} & -1.715996 & -0.164390 & 0.070651 \\ \mathrm{C} & -0.250107 & -0.833164 & 0.237599 \\ \mathrm{SI} & 1.476668 & -0.043162 & -0.013922 \\ \mathrm{~N} & -2.041999 & 1.428004 & 0.017532 \\ \mathrm{H} & -1.358688 & 2.096375 & 0.360862 \\ \mathrm{H} & -2.714822 & 1.789028 & -0.653607 \\ \mathrm{~N} & -3.148156 & -0.930948 & -0.068106 \\ \mathrm{H} & -3.184993 & -1.890375 & -0.399511 \\ \mathrm{H} & -3.951772 & -0.621922 & 0.473217 \\ \mathrm{C} & 1.304071 & 1.824386 & -0.104380 \\ \mathrm{H} & 0.904911 & 2.253572 & 0.821073 \\ \mathrm{H} & 2.298072 & 2.262631 & -0.239367 \\ \mathrm{H} & 0.700205 & 2.158008 & -0.953357 \\ \mathrm{C} & 2.468040 & -0.565095 & 1.480846 \\ \mathrm{H} & 2.542385 & -1.653638 & 1.556633 \\ \mathrm{H} & 3.487452 & -0.172309 & 1.413684 \\ \mathrm{H} & 2.029406 & -0.190540 & 2.409805 \\ \mathrm{C} & 2.150490 & -0.742291 & -1.611330 \\ \mathrm{H} & 3.165491 & -0.371495 & -1.786207 \\ \mathrm{H} & 2.200127 & -1.834341 & -1.585059 \\ \mathrm{H} & 1.539152 & -0.452355 & -2.470518 \\ \mathrm{H} & -0.294207 & -1.884920 & 0.525104\end{array}$




$\begin{array}{crrr}47 \mathrm{H} & & & \\ \mathrm{P} & -0.882567 & -0.188332 & 0.223617 \\ \mathrm{C} & 0.565832 & -0.879558 & 0.522886 \\ \mathrm{SI} & 2.279510 & -0.258817 & -0.029015 \\ \mathrm{~N} & -1.112399 & 1.395663 & -0.048801 \\ \mathrm{~N} & -2.289008 & -0.997733 & 0.079824 \\ \mathrm{C} & 2.058849 & 0.790838 & -1.569364 \\ \mathrm{H} & 1.470416 & 1.694812 & -1.392480 \\ \mathrm{H} & 3.037288 & 1.113201 & -1.939053 \\ \mathrm{H} & 1.580481 & 0.222204 & -2.372452 \\ \mathrm{C} & 3.083475 & 0.706511 & 1.361501 \\ \mathrm{H} & 3.125848 & 0.118987 & 2.282813 \\ \mathrm{H} & 4.111949 & 0.964126 & 1.088783 \\ \mathrm{H} & 2.561789 & 1.641667 & 1.581544 \\ \mathrm{C} & 3.237545 & -1.821058 & -0.407758 \\ \mathrm{H} & 4.249350 & -1.573523 & -0.743745 \\ \mathrm{H} & 3.333803 & -2.458875 & 0.475745 \\ \mathrm{H} & 2.760828 & -2.406319 & -1.198970 \\ \mathrm{C} & -2.310028 & -2.409635 & -0.335400 \\ \mathrm{H} & -3.130207 & -2.547882 & -1.043811 \\ \mathrm{H} & -1.368996 & -2.668661 & -0.821158 \\ \mathrm{H} & -2.465320 & -3.061310 & 0.528633 \\ \mathrm{C} & -3.560806 & -0.481634 & 0.610989 \\ \mathrm{H} & -3.438044 & 0.546371 & 0.950650 \\ \mathrm{H} & -4.317703 & -0.513820 & -0.176268 \\ \mathrm{H} & -3.885255 & -1.101381 & 1.451452 \\ \mathrm{C} & -1.940736 & 1.896546 & -1.156972 \\ \mathrm{H} & -2.722354 & 2.550697 & -0.762546 \\ \mathrm{H} & -1.311421 & 2.461380 & -1.850301 \\ \mathrm{H} & -2.401377 & 1.064068 & -1.688592 \\ \mathrm{C} & -0.426961 & 2.429295 & 0.742565 \\ \mathrm{H} & 0.110510 & 1.969459 & 1.571591 \\ \mathrm{H} & 0.278295 & 2.980119 & 0.115488 \\ \mathrm{H} & -1.175059 & 3.122335 & 1.137253 \\ \mathrm{H} & 0.487375 & -1.842585 & 1.029532 \\ & & & \\ \mathrm{C} & -0.125133 & 3.830656 & -0.636699 \\ \mathrm{C} & -0.511403 & 3.636404 & 0.695787 \\ \mathrm{C} & 0.000000 & 1.271646 & 0.467803 \\ \mathrm{C} & 0.356275 & 2.763954 & -1.409114 \\ \mathrm{H} & 4.827027 & -1.068926 \\ \mathrm{H} & -4.476176 & 1.289233\end{array}$




\begin{tabular}{|c|c|c|c|}
\hline $\mathrm{H}$ & -0.711309 & 0665 & 19 \\
\hline $\mathrm{H}$ & 0.700868 & 2.944138 & -2.423077 \\
\hline $\mathrm{C}$ & 0.000000 & 0.000000 & 1.079358 \\
\hline $\mathrm{C}$ & 0.000000 & -1.271646 & 0.467803 \\
\hline $\mathrm{C}$ & 0.420223 & -2.375283 & 1.259662 \\
\hline $\mathrm{C}$ & 0.511403 & -3.636404 & 0.695787 \\
\hline $\mathrm{C}$ & 0.125133 & -3.830656 & -0.63 \\
\hline $\mathrm{C}$ & -0.356275 & -2.763954 & -1.409114 \\
\hline $\mathrm{C}$ & -0.418813 & -1.488578 & -0.873704 \\
\hline $\mathrm{H}$ & 1309 & -2.210665 & 2.294919 \\
\hline $\mathrm{H}$ & 0.859973 & -4.4 & 233 \\
\hline $\mathrm{H}$ & 0.171503 & -4.827027 & -1.068926 \\
\hline $\mathrm{H}$ & -0.700868 & -2.9 & -2.42 \\
\hline $\mathrm{H}$ & -0.869150 & -0.6 & -1.439005 \\
\hline $\mathrm{H}$ & 0.86 & 0.6 & -1.4 \\
\hline $\mathrm{H}$ & 000 & 00 & 35 \\
\hline \multicolumn{4}{|c|}{$49 \mathrm{H}$} \\
\hline $\mathrm{C}$ & -0.0 & -1.3 & 0.0 \\
\hline $\mathrm{C}$ & -0.6 & 1.3 & \\
\hline $\mathrm{C}$ & 713 & 1.31 & -0.06 \\
\hline $\mathrm{H}$ & -1.2 & 2.2 & 38 \\
\hline $\mathrm{H}$ & 1.289953 & 2.21 & -0.0 \\
\hline $\mathrm{P}$ & -1.380288 & -0.291 & -0.10 \\
\hline $\mathrm{P}$ & 069 & -0.29 & 0.10 \\
\hline $\mathrm{H}$ & & & \\
\hline $\mathrm{H}$ & 2.4 & -0.5 & -0.71 \\
\hline $\mathrm{H}$ & -0.000074 & -2.3 & 765 \\
\hline \multicolumn{4}{|l|}{$50 \mathrm{H}$} \\
\hline $\mathrm{C}$ & 000 & 0.0 & 41 \\
\hline $\mathrm{C}$ & 0.000000 & 0.7 & -1.28 \\
\hline $\mathrm{C}$ & 0.000000 & -0.76 & -1.284001 \\
\hline $\mathrm{H}$ & -0.601699 & 1.16 & -2.10 \\
\hline $\mathrm{H}$ & & & \\
\hline $\mathrm{P}$ & -0.7 & 1.229 & 0.35 \\
\hline $\mathrm{P}$ & 0.7 & -1.229 & 0.35 \\
\hline $\mathrm{H}$ & -0.327597 & 2.498 & 0.80 \\
\hline $\mathrm{H}$ & 0.327597 & -2.49 & 0.806853 \\
\hline $\mathrm{H}$ & -1.019166 & -1.164869 & -1.360066 \\
\hline $\mathrm{H}$ & & 1.164869 & -1.360066 \\
\hline $\mathrm{H}$ & 0.000000 & 0.000000 & 2.391103 \\
\hline \multicolumn{4}{|l|}{$51 \mathrm{H}$} \\
\hline $\mathrm{C}$ & 0.000000 & 0.70 & 0.215724 \\
\hline $\mathrm{C}$ & 0.000000 & -0.702610 & 0.215724 \\
\hline
\end{tabular}




$\begin{array}{crrr}\mathrm{C} & 0.000000 & -1.444540 & -0.969709 \\ \mathrm{C} & 0.000000 & -0.707345 & -2.143193 \\ \mathrm{C} & 0.000000 & 0.707345 & -2.143193 \\ \mathrm{C} & 0.000000 & 1.444540 & -0.969709 \\ \mathrm{C} & 0.000000 & 0.000000 & 2.332094 \\ \mathrm{H} & 0.000000 & -2.530498 & -0.973982 \\ \mathrm{H} & 0.000000 & 2.530498 & -0.973982 \\ \mathrm{H} & 0.000000 & 2.039620 & 1.899447 \\ \mathrm{H} & 0.000000 & -2.039620 & 1.899447 \\ \mathrm{~N} & 0.000000 & 1.083592 & 1.549864 \\ \mathrm{~N} & 0.000000 & -1.083592 & 1.549864 \\ \mathrm{H} & 0.000000 & -1.231191 & -3.094329 \\ \mathrm{H} & 0.000000 & 1.231191 & -3.094329 \\ \mathrm{H} & 0.000000 & 0.000000 & 3.413204 \\ & & & \\ 52 \mathrm{H} & & & \\ \mathrm{C} & 1.232254 & 0.331741 & 0.107716 \\ \mathrm{C} & 0.057440 & 1.224338 & -0.047150 \\ \mathrm{C} & -0.866274 & -0.906799 & 0.115334 \\ \mathrm{C} & 0.636957 & -1.067654 & -0.147082 \\ \mathrm{H} & 2.043240 & 0.606797 & -0.572110 \\ \mathrm{H} & 1.620019 & 0.456250 & 1.128716 \\ \mathrm{H} & -1.965045 & 1.003971 & -0.107942 \\ \mathrm{H} & -1.169074 & -1.162026 & 1.134588 \\ \mathrm{H} & -1.510033 & -1.428792 & -0.593726 \\ \mathrm{H} & 1.077406 & -1.830289 & 0.495416 \\ \mathrm{H} & 0.804296 & -1.359754 & -1.185617 \\ \mathrm{~N} & -1.045603 & 0.559318 & -0.050043 \\ \mathrm{H} & 0.056144 & 2.308858 & -0.121937\end{array}$

